$\underline{\text { Preprint typeset in JHEP style. - HYPER VERSION }}$

Cavendish-HEP-03/17

CERN-TH/2003-205

\title{
Hawking Radiation from a $(4+n)$-dimensional Black Hole: Exact Results for the Schwarzschild Phase
}

\author{
C.M. Harris ${ }^{a}$ and P. Kanti ${ }^{b}$ \\ ${ }^{a}$ Cavendish Laboratory, University of Cambridge, Madingley Road, Cambridge, \\ CBз OHE, UK. \\ ${ }^{b}$ Theory Division, CERN, 1211 Geneva 23, Switzerland.
}

\begin{abstract}
We start our analysis by deriving a master equation that describes the motion of a field with arbitrary spin $s$ on a 3-brane embedded in a non-rotating, uncharged $(4+n)$-dimensional black hole background. By numerical analysis, we derive exact results for the greybody factors and emission rates for scalars, fermions and gauge bosons emitted directly on the brane, for all energy regimes and for an arbitrary number $n$ of extra dimensions. The relative emissivities on the brane for different types of particles are computed and their dependence on the dimensionality of spacetime is demonstrated - we therefore conclude that both the amount and the type of radiation emitted can be used for the determination of $n$ if the Hawking radiation from these black holes is observed. The emission of scalar modes in the bulk from the same black holes is also studied and the relative bulk-to-brane energy emissivity is accurately computed. We demonstrate that this quantity varies considerably with $n$ but always remains smaller than unity - this provides firm support to earlier arguments made by Emparan, Horowitz and Myers.
\end{abstract}

Keywords: Beyond Standard Model, Black Holes, Large Extra Dimensions. 


\section{Contents}

1. Introduction 1

2. Basic Formulae and Assumptions 4

3. Greybody factors for Emission on the Brane 6

3.1 Analytic calculation 9

3.2 Numerical calculation 9

4. Numerical Results for Brane Emission 11

4.1 Spin 0 fields 12

4.2 Spin $1 / 2$ fields 14

4.3 Spin 1 fields 16

4.4 Relative Emissivities for different species 18

5. Emission in the Bulk 20

5.1 Greybody Factors and Emission Rates 21

5.2 Bulk-to-Brane Relative Emissivities 25

6. Conclusions 27

\section{Introduction}

Motivated by the desire to explain the hierarchy problem — that is, the sixteen orders of magnitude difference between the electroweak energy scale and the Planck scale - models that postulate the existence of extra dimensions have been revived and extensively studied during the last few years after the work by Arkani-Hamed, Dimopoulos and Dvali (ADD) [1] and Randall and Sundrum (RS) [2] (for some early works, see [3]). In the standard version of those works the Standard Model fields are localized on a 3-brane, which plays the role of our 4-dimensional world, while gravity can propagate both on the brane and in the bulk - the spacetime transverse to the brane. In theories with large extra dimensions the traditional Planck scale, $M_{P l} \sim 10^{18} \mathrm{GeV}$, is only an effective energy scale derived from the fundamental higher-dimensional one, $M_{*}$, through the following relation [1]

$$
M_{P l}^{2} \sim M_{*}^{n+2} R^{n}
$$


The above relation involves the volume of the extra dimensions, $V \sim R^{n}$, under the assumption that $R$ is the common size of all $n$ extra compact dimensions. Therefore, if the volume of the internal space is large (i.e. if $R \gg \ell_{P l}$, where $\ell_{P l}=10^{-33} \mathrm{~cm}$ is the Planck length) then $M_{*}$ can be substantially lower than $M_{P l}$.

In the regime $r \ll R$, the extra dimensions 'open up' and gravity becomes strong. Hence, Newton's law for the gravitational interactions in this regime is modified, with the gravitational potential assuming a $1 / r^{n+1}$ dependence on the radial separation between two massive particles. Experiments which measure the gravitational inversesquare law at short scales can provide limits on the size of the extra dimensions, or equivalently on the value of the fundamental scale $M_{*}$; for $n=2$ such measurements give $M_{*}>3.5 \mathrm{TeV}$ [4] (the $n=1$ case has already been excluded by astronomical data). Since gravitons can propagate both in the bulk and on the brane, massive Kaluza-Klein (KK) graviton states can modify both the cross sections of Standard Model particle interactions and astrophysical or cosmological processes. The absence of signatures of production of either real or virtual KK gravitons at colliders puts a relatively weak lower limit on $M_{*}$ - from $1.45 \mathrm{TeV}$ (for $\left.n=2\right)$ to $0.6 \mathrm{TeV}(n=6)$ [5]. Much more stringent constraints arise if one considers astrophysical or cosmological processes; ignoring the systematic errors, these constraints exclude by far even the $n=3$ case, while allowing models with $M_{*} \sim 1 \mathrm{TeV}$ and $n \geq 4$ [6].

If extra dimensions with $R \gg \ell_{P l}$ exist, then black holes with a horizon radius $r_{H}$ smaller than the size of the extra dimensions $R$ are virtually higher-dimensional objects centered on the brane and extending along the extra dimensions. It has been shown that these small black holes have modified properties, i.e. they are larger and colder compared to a 4-dimensional black hole with exactly the same mass $M_{B H}$ [7]. One striking consequence of the theories with large extra dimensions is that the lowering of the fundamental gravity scale allows for the production of such miniature black holes during high energetic scattering processes with centre-ofmass energy $\sqrt{s} \gg M_{*}$ [8-14]. Arguments based on Thorne's hoop conjecture [15] predict the creation of a black hole in the case where any two partons of the colliding particles pass within the horizon radius corresponding to their centre-of-mass energy. The black holes created will have a mass equal to a fraction of $\sqrt{s}$ varying from 0.84 down to 0.58 depending on the details of the scattering process and the number of extra dimensions [16]. Provided that the mass of the black hole is larger than a few times the fundamental Planck mass, these objects could still be treated semiclassically. Miniature black holes may be created in the atmosphere of the earth (during scattering processes of cosmic rays) and at future particle colliders, if the fundamental energy scale is low enough. Several aspects of these processes have been studied in Refs. [17-22].

Once produced, these miniature black holes are expected to decay almost instantaneously (typical lifetimes are $\sim 10^{-26} \mathrm{~s}$ ). According to Refs. $[9,13]$, the produced black holes will go through a number of phases before completely evaporating. These 
are:

- The balding phase: the black hole emits mainly gravitational radiation and sheds the 'hair' inherited from the original particles, and the asymmetry due to the violent production process.

- The spin-down phase: the typically non-zero impact parameter of the colliding partons leads to black holes with some angular momentum about an axis perpendicular to the plane. During this phase, the black hole loses its angular momentum through the emission of Hawking radiation [23] and, possibly, through superradiance.

- The Schwarzschild phase: a spherically-symmetric black hole loses energy due to the emission of Hawking radiation. This results in the gradual decrease of its mass and the increase of its temperature.

- The Planck phase: the mass and/or the Hawking temperature approach the Planck scale - a theory of quantum gravity is necessary to study this phase in detail.

As in the 4-dimensional case [24], it is reasonable to expect that the Schwarzschild phase in the life of a small higher-dimensional black hole will be the longer one, and will account for the greatest proportion of the mass loss through the emission of Hawking radiation. Throughout this paper, we will focus on the emission of energy, in both brane-localized and bulk modes, from a non-rotating, uncharged $(4+n)$ dimensional black hole. Initially we will consider the emission of particle modes on the brane, which is the most phenomenologically interesting effect since it involves Standard Model particles. We will start by deriving a master equation describing the motion of a field with arbitrary spin $s$ in the spherically-symmetric black hole background induced on the brane - this will help resolve ambiguities present in similar equations which have previously appeared in the literature. We will then produce exact numerical results for the greybody factors and emission rates for each type of particle and for an arbitrary number $n$ of extra dimensions, and compare them with those derived from earlier analytic studies of the Schwarzschild phase [25,26]. We will show that the full analytic results derived in [26] closely follow the exact results in the low- and intermediate-energy regimes while the power expansion employed in [25] can accurately describe only a very limited low-energy regime, especially in the case of scalar fields. The major aim of this study is to demonstrate the dependence of the above quantities on the dimensionality of spacetime in all energy regimes. This might serve as a tool for 'reading' the number of extra dimensions existing in nature if the spectrum of Hawking radiation emitted by these small black holes is detected. In the framework of the same analysis, we will also derive the relative emissivities for 
scalars, fermions and gauge bosons and demonstrate how these change as the number of extra dimensions projected onto the brane varies - a potentially additional signature to be used in the determination of $n$.

In Ref. [27], it was argued that the majority of energy during the emission of Hawking radiation from a higher-dimensional black hole is emitted into modes on the brane (i.e. Standard Model fermions and gauge bosons, zero-mode gravitons and scalar fields). This argument was based on their result that a single zero-mode brane particle carries as much energy as the whole Kaluza-Klein (KK) tower of massive excitations propagating in the bulk - the abundance of brane modes compared to bulk modes then leads to the aforementioned conclusion. In this paper, we also study the emission of bulk scalar modes from a $(4+n)$-dimensional black hole, and compare the total bulk and brane emissivities in an attempt to confirm or disprove the above argument. Our analysis improves the heuristic arguments made in [27] and for the first time provides exact and detailed results concerning the relative bulk and brane emissivities in all energy regimes and for various values of $n$.

We start our analysis in section 2 by presenting the basic formulae and assumptions for the gravitational background and emission rates from small higherdimensional black holes. In section 3 , we present a master equation describing the motion of scalars, fermions and gauge bosons in the 4-dimensional induced background - the basic steps of this calculation are given in the Appendix; in the same section, we also define the corresponding greybody factor and emission rates and discuss analytic and numerical methods for computing these quantities. Exact numerical results for emission on the brane (for scalars, fermions and gauge bosons) are presented in section 4, as well as a discussion of the relative emissivities and their dependence on $n$. The emission of bulk scalar modes is thoroughly studied in section 5 , and our results for the relative bulk-to-brane emissivities are also presented in this section. Our conclusions are summarized in section 6 .

\section{Basic Formulae and Assumptions}

Let us start with the form of the gravitational background around a non-rotating, uncharged $(4+n)$-dimensional Schwarzschild black hole. The line-element is given by [28]

$$
d s^{2}=-h(r) d t^{2}+h(r)^{-1} d r^{2}+r^{2} d \Omega_{2+n}^{2}
$$

where

$$
h(r)=1-\left(\frac{r_{H}}{r}\right)^{n+1}
$$

and with the angular part given by

$$
d \Omega_{2+n}^{2}=d \theta_{n+1}^{2}+\sin ^{2} \theta_{n+1}\left(d \theta_{n}^{2}+\sin ^{2} \theta_{n}\left(\ldots+\sin ^{2} \theta_{2}\left(d \theta_{1}^{2}+\sin ^{2} \theta_{1} d \varphi^{2}\right) \ldots\right)\right) .
$$


In the above, $0<\varphi<2 \pi$ and $0<\theta_{i}<\pi$, for $i=1, \ldots, n+1$. By using an analogous approach to the usual 4-dimensional Schwarzschild calculation, i.e. by applying Gauss' law in the $(4+n)$-dimensional spacetime, we obtain the following horizon radius

$$
r_{H}=\frac{1}{\sqrt{\pi} M_{*}}\left(\frac{M_{B H}}{M_{*}}\right)^{\frac{1}{n+1}}\left(\frac{8 \Gamma\left(\frac{n+3}{2}\right)}{n+2}\right)^{\frac{1}{n+1}},
$$

where $M_{B H}$ is the mass of the black hole. The black holes being considered in this work are assumed to have horizon radii satisfying the relation $\ell_{P l} \ll r_{H} \ll R$. The former inequality guarantees that no quantum corrections are important in our calculations, while the latter is necessary for these black holes to be considered as higher-dimensional objects. The tension of the brane on which the black hole is centered is assumed to be much smaller than the black hole mass and thus it can be ignored in our analysis.

A black hole of a particular horizon radius $r_{H}$ is characterized by a Hawking temperature related by the expression

$$
T_{B H}=\frac{(n+1)}{4 \pi r_{H}} .
$$

The above temperature gives rise to almost blackbody radiation. The flux spectrum, i.e. the number of particles emitted per unit time, is given by [23]

$$
\frac{d N^{(s)}(\omega)}{d t}=\sum_{\ell} \sigma_{\ell}^{(s)}(\omega) \frac{1}{\exp \left(\omega / T_{B H}\right) \pm 1} \frac{d^{n+3} k}{(2 \pi)^{n+3}},
$$

while the power spectrum, i.e. the energy emitted per unit time, is

$$
\frac{d E^{(s)}(\omega)}{d t}=\sum_{\ell} \sigma_{\ell}^{(s)}(\omega) \frac{\omega}{\exp \left(\omega / T_{B H}\right) \pm 1} \frac{d^{n+3} k}{(2 \pi)^{n+3}} .
$$

In the above, $s$ is the spin of the degree of freedom being considered and $\ell$ is the angular momentum quantum number. The spin statistics factor in the denominator is -1 for bosons and +1 for fermions. For massless particles, $|k|=\omega$ and the phasespace integral reduces to an integral over $\omega$. The term in front, $\sigma_{\ell}^{(s)}(\omega)$, is the socalled 'greybody' factor ${ }^{1}$ which encodes valuable information for the structure of the spacetime around the black hole (which emits the Hawking radiation) including the dimensionality of spacetime. This quantity can be determined by solving the equation of motion of a particular degree of freedom in the aforementioned background and computing the corresponding absorption coefficient $\mathcal{A}_{\ell}^{(s)}$. Then, we may write [29]

$$
\sigma_{\ell}^{(s)}(\omega)=\frac{2^{n} \pi^{(n+1) / 2} \Gamma[(n+1) / 2]}{n ! \omega^{n+2}} \frac{(2 \ell+n+1)(\ell+n) !}{\ell !}\left|\mathcal{A}_{\ell}^{(s)}\right|^{2} .
$$

\footnotetext{
${ }^{1}$ The quantity $\sigma_{\ell}^{(s)}(\omega)$ is alternatively called the absorption cross section. It is also common in the literature to refer to the absorption probability $\left|\mathcal{A}_{\ell}^{(s)}\right|^{2}$, related to $\sigma_{\ell}^{(s)}(\omega)$ through Eq. (2.8), as the greybody factor.
} 
As the decay progresses, the black hole mass decreases and the Hawking temperature rises. It is usually assumed that a quasi-stationary approach to the decay is valid that is, the black hole has time to come into equilibrium at each new temperature before the next particle is emitted. We will make this assumption also here.

We would like finally to stress that Eqs. (2.6) and (2.7) refer to individual degrees of freedom and not to elementary particles, like electrons or quarks, which contain more than one polarization. Combining the necessary degrees of freedom and their corresponding flux or power spectra, the number of elementary particles produced, and the energy they carry, can be easily computed. For more information on this, we refer the reader to Ref. [30] where a Black Hole Event Generator has been constructed. This simulates both the production and decay of small black holes at hadronic colliders and, by using the results for the greybody factors derived here, provides estimates for the number and spectra of the different types of elementary particles produced.

\section{Greybody factors for Emission on the Brane}

The greybody factors modify the spectrum of emitted particles from that of a perfect thermal blackbody even in four dimensions [23]. For a 4-dimensional Schwarzschild black hole, geometric arguments show that, in the high-energy regime, $\Sigma_{l}\left|\mathcal{A}_{\ell}\right|^{2} \propto$ $\left(\omega r_{H}\right)^{2}$ which means that the greybody factor at high energies is independent of $\omega$ and the spectrum is exactly like that of a blackbody for every particle species $[24,31,32]$. The low-energy behaviour, on the other hand, is strongly spin-dependent. A common feature for fields with spin $s=0, \frac{1}{2}$ and 1 is that the greybody factors reduce the low-energy emission rate significantly below the geometrical optics value $[24,33]$. The result is that both the power and flux spectra peak at higher energies than those for a blackbody at the same temperature. The spin dependence of the greybody factors means that they are necessary to determine the relative emissivities of different particle types from a black hole (at present these are only available in the literature for the $4 \mathrm{D}$ case $[24,32])$.

The procedure for calculating greybody factors needs to be generalised to include emission from small higher-dimensional black holes with $r_{H}<R$ which emit radiation either in the bulk or on the brane. Analytical studies [25,26] have shown that the greybody factors in that case have a strong dependence on the number of extra dimensions. The dependence on both energy and the number of dimensions means that the exact form of the greybody factors should be taken into account in any attempt to determine the number of extra dimensions by studying the energy spectrum of particles emitted from a higher-dimensional black hole. In this section, as well as in section 4, we will focus on the emission of brane-localised modes leaving the study of bulk emission and of relative bulk-to-brane emissivity for section 5 . 
The brane-localised modes propagate in a 4-dimensional black hole background which is the projection of the higher-dimensional one, given in Eq. (2.1), onto the brane. The induced metric tensor follows by fixing the values of the extra angular coordinates, $\theta_{i}=\pi / 2$ for $i \geq 2$, and it is found to have the form

$$
d s^{2}=-h(r) d t^{2}+h(r)^{-1} d r^{2}+r^{2}\left(d \theta^{2}+\sin ^{2} \theta d \varphi^{2}\right) .
$$

The greybody factors are determined from the amplitudes of in-going and outgoing waves at infinity so the essential requirement is to solve the equation of motion of a particle propagating in the above background. For this purpose, we have derived in the Appendix a generalised master equation for a particle with arbitrary spin $s$, similar to the one derived by Teukolsky [34]. For $s=\frac{1}{2}$ and 1, the equation of motion has been derived by using the Newman-Penrose method [35,36], while for $s=0$ the corresponding equation follows by the evaluation of the double covariant derivative $g^{\mu \nu} D_{\mu} D_{\nu}$ acting on the scalar field. The derived master equation is separable in each case and, by using the factorization

$$
\Psi_{s}=e^{-i \omega t} e^{i m \varphi} R_{s}(r) S_{s, l}^{m}(\theta),
$$

we obtain the radial equation

$$
\Delta^{-s} \frac{d}{d r}\left(\Delta^{s+1} \frac{d R_{s}}{d r}\right)+\left(\frac{\omega^{2} r^{2}}{h}+2 i \omega s r-\frac{i s \omega r^{2} h^{\prime}}{h}+s\left(\Delta^{\prime \prime}-2\right)-\lambda_{s l}\right) R_{s}(r)=0,
$$

where $\Delta=h r^{2}$. The corresponding angular equation has the form

$$
\frac{1}{\sin \theta} \frac{d}{d \theta}\left(\sin \theta \frac{d S_{s, l}^{m}}{d \theta}\right)+\left(-\frac{2 m s \cot \theta}{\sin \theta}-\frac{m^{2}}{\sin ^{2} \theta}+s-s^{2} \cot ^{2} \theta+\lambda_{s l}\right) S_{s, l}^{m}(\theta)=0,
$$

where $Y_{s, l}^{m}=e^{i m \varphi} S_{s, l}^{m}(\theta)$ are known as the spin-weighted spherical harmonics and $\lambda_{s l}$ is a separation constant which is found to have the value $\lambda_{s l}=l(l+1)-s(s+1)$ [37]. For $s=0$, Eq. (3.3) reduces as expected to Eq. (41) of Ref. [25] which was used for the analytical study of the emission of brane-localised scalar modes from a spherically-symmetric higher-dimensional black hole. Under the redefinition $R_{s}=\Delta^{-s} P_{s}$, Eq. (3.3) assumes a form similar to that of Eq. (11) of Ref. [26] which was used for the study of brane-localised fermion and gauge boson emission. The two equations differ due to an extra term in the expression of the latter one, which although vanishing for $s=\frac{1}{2}$ and 1 (thus leading to the correct results for the greybody factors for fermion and gauge boson fields) gives a non-vanishing contribution for all other values of $s$. Therefore, the generalized equation derived by Cvetic and Larsen [38] can not be considered as a master equation valid for all types of fields. A similar equation was derived in Ref. [39] but due to a typographical error the multiplicative factor $s$ in front of the $\Delta^{\prime \prime}$-term is missing, thus leading to an apparently 
different radial equation for all values of $s$. Therefore, before addressing the question of the derivation of the exact forms of the greybody factors in the brane background, the derivation of a consistent master equation was imperative. This task was indeed performed, with some of the steps of the calculation presented in the Appendix, and has led to the aforementioned Eqs. (3.3) and (3.4).

For the derivation of the greybody factors associated with the emission of fields from the projected black hole, we need to know the asymptotic solutions of (3.3) both as $r \rightarrow r_{H}$ and as $r \rightarrow \infty$. In the former case, the solution is of the form

$$
R_{s}^{(h)}=A_{i n}^{(h)} \Delta^{-s} e^{-i \omega r^{*}}+A_{\text {out }}^{(h)} e^{i \omega r^{*}},
$$

where

$$
\frac{d r^{*}}{d r}=\frac{1}{h(r)}
$$

We impose the boundary condition that there is no out-going solution near the horizon of the black hole, and therefore set $A_{\text {out }}^{(h)}=0$. The solution at infinity is of the form

$$
R_{s}^{(\infty)}=A_{i n}^{(\infty)} \frac{e^{-i \omega r}}{r}+A_{\text {out }}^{(\infty)} \frac{e^{i \omega r}}{r^{2 s+1}},
$$

and comprises both in-going and out-going modes.

The greybody factor $\sigma_{\ell}(\omega)$ for the emission of brane-localised modes is related to the energy absorption coefficient $\mathcal{A}_{\ell}$ through the simplified relation

$$
\hat{\sigma}_{\ell}^{(s)}(\omega)=\frac{\pi}{\omega^{2}}(2 \ell+1)\left|\hat{\mathcal{A}}_{\ell}^{(s)}\right|^{2},
$$

where henceforth quantities with a 'hat' will denote quantities associated with the emission of brane-localised modes. The above relation follows from Eq. (2.8) by setting $n=0$ since the emission of brane-localised modes is a 4-dimensional process. In the same way, the power spectrum of the Hawking radiation emitted on the brane can be computed by the 4-dimensional expression

$$
\frac{d \hat{E}^{(s)}(\omega)}{d t}=\sum_{\ell} \hat{\sigma}_{\ell}^{(s)}(\omega) \frac{\omega}{\exp \left(\omega / T_{B H}\right) \pm 1} \frac{d^{3} k}{(2 \pi)^{3}} .
$$

Note, however, that the absorption coefficient $\hat{\mathcal{A}}_{\ell}$ still depends on the number of extra dimensions since the projected metric tensor (3.1) carries a signature of the dimensionality of the bulk spacetime through the expression of the metric function $h(r)$. The absorption coefficient itself is defined as

$$
\left|\hat{\mathcal{A}}_{\ell}^{(s)}\right|^{2}=1-\frac{\mathcal{F}_{\text {out }}^{(\infty)}}{\mathcal{F}_{\text {in }}^{(\infty)}}=\frac{\mathcal{F}_{\text {in }}^{(h)}}{\mathcal{F}_{\text {in }}^{(\infty)}},
$$

in terms of the energy fluxes evaluated either at infinity or at the horizon. The two definitions are related by simple energy conservation and lead to the same results. Both of them may be used for the determination of the absorption coefficient, depending on the type of particle emitted and the method of the study, numerical or analytic, which is followed. 


\subsection{Analytic calculation}

The greybody factors have been determined analytically for the 4-dimensional case in Refs. [24,32] both for a rotating and non-rotating black hole. In the $(4+n)$ dimensional case, Refs. [25, 26] have provided analytic expressions for the greybody factors for the emission of scalars, fermions and gauge bosons from a higherdimensional Schwarzschild-like black hole emitting radiation both in the bulk and on the brane, and for an arbitrary number of extra dimensions $n$. Ref. [39] presented analytic results for the greybody factors for the emission of brane-localized modes from a Kerr-like black hole in the particular case of $n=1$. All the above results were derived in the low-energy approximation where the procedure used is as follows:

- Find an analytic solution in the near-horizon regime and expand as in-going and out-going waves so that the $A^{(h)}$ coefficients can be extracted.

- Apply the boundary condition on the horizon so that the wave is purely outgoing.

- Find an analytic solution in the far-field regime and again expand as in-going and out-going waves.

- Match the two solutions in the intermediate regime.

- Extract $\mathcal{A}_{\ell}$ and expand in powers of $\left(\omega r_{H}\right)$.

The solution obtained by following the above approximate method is a power series in $\omega r_{H}$, which is only valid for low energies and expected to significantly deviate from the exact result as the energy of the emitted particle increases (this was pointed out in $[24,33]$ for the 4-dimensional case). In [26], the full analytic result for the absorption coefficient (before the final expansion was made) was used for the evaluation of the emission rates for all particle species. The range of validity of these results, although improved compared to the power series, was still limited since the assumption of small $\omega r_{H}$ was still made during the matching of the two solutions in the intermediate regime. As the equations of motion for all types of particles are too cumbersome to solve analytically for any value of $\omega r_{H}$, it becomes clear that only an exact numerical analysis can yield the full results for greybody factors and emission rates.

\subsection{Numerical calculation}

There are various numerical issues which arise while trying to do the full calculation of the absorption coefficient; the complexity of these problems strongly depends on the spin $s$ of the emitted particle. The usual numerical procedure starts by applying the required horizon boundary condition (a vanishing out-going wave) to $R_{s}(r)$. Then, 
the solution is integrated out to 'infinity' and the asymptotic coefficient $A_{\text {in }}^{(\infty)}$ is extracted in terms of which $\mathcal{A}_{\ell}$ can be calculated.

By looking at the asymptotic solution at infinity, Eq. (3.7), one can see that, for the scalar case, the in-going and out-going waves are of comparable magnitude. Therefore it is relatively easy to extract the coefficients at infinity and thus determine the greybody factor. However for fields with non-vanishing spin, i.e. fermions, vector bosons and gravitons, this is not an easy task. First of all, different components carry a different part of the emitted field: the upper component $\Psi_{+s}$ consists mainly of the in-going wave with the out-going one being greatly suppressed, while for the lower component $\Psi_{-s}$ the situation is reversed (for a field with spin $s \neq 0$, only the upper and lower components are radiative). Distinguishing between the two parts of the solution, in-going and out-going, is not an easy task no matter which component we look at, and it becomes more difficult as the magnitude of the spin increases. In addition, the choice of either positive or negative $s$ to extract the greybody factor, i.e. using either the upper or the lower component, affects the numerical issues.

If $s$ is negative, then the horizon boundary condition is easy to apply because components of the in-going solution on the horizon will be exponentially suppressed [see Eq. (3.5) above]. However, negative $s$ also means that at infinity the out-going solution is enhanced by $r^{-2 s}$ with respect to the in-going one [see Eq. (3.7)], which makes accurate determination of $A_{i n}^{(\infty)}$ very difficult. Hence the negative $s$ approach is not used in this work.

On the other hand if $s$ is positive, it is easy to accurately extract $A_{\text {in }}^{(\infty)}$ because the out-going solution at infinity is suppressed by a factor of $r^{-2 s}$. However, close to the horizon the out-going solution is exponentially smaller than the in-going one. This means that the solution for $R_{s}(r)$ can be easily contaminated by components of the out-going solution. This problem becomes worse for larger $s$ and first becomes significant for $s=1$.

For $n=0$, various methods (see e.g. [40]) have been used to solve the numerical problems which arise in the gauge boson case. The approach of Bardeen in [40] is also applicable for $n=1$, but no analogous method was found for higher numbers of extra dimensions. Here, an alternative transformation of the radial equation was used instead. Writing $y=r / r_{H}$ and $R_{1}=y F(y) e^{-i \omega r^{*}}$, the wave equation becomes

$$
\left(h y^{2}\right) \frac{d^{2} F}{d y^{2}}+2 y\left(h-i \omega r_{H} y\right) \frac{d F}{d y}-l(l+1) F=0 .
$$

Since on the horizon $y=1$ and $h=0$, the boundary conditions become $F(1)=1$ and

$$
\left.\frac{d F}{d y}\right|_{y=1}=\frac{i l(l+1)}{2 \omega r_{H}} .
$$

For fermions, no such transformation was necessary and so the radial equation of Eq. (3.3) was used. However the application of the boundary condition at the 
horizon is made slightly easier by the transformation $P_{s}=\Delta^{s} R_{s}$, which means that the asymptotic solution at the horizon becomes

$$
P_{s}^{(h)}=A_{\text {in }}^{(h)} e^{-i \omega r^{*}}+A_{\text {out }}^{(h)} \Delta^{s} e^{i \omega r^{*}} .
$$

Since we require $A_{\text {out }}^{(h)}=0$, suitable boundary conditions to apply, when solving the differential equation for $P_{s}$, are that, as $r \rightarrow r_{H}$,

$$
P_{s}=1
$$

while using Eq. (3.6), we also obtain

$$
\frac{d P_{s}}{d r}=-i \omega \frac{d r *}{d r}=-\frac{i \omega}{h(r)} .
$$

The above boundary conditions ensure that $\left|A_{i n}^{(h)}\right|^{2}=1$. The asymptotic form for $P_{s}$ at infinity now looks like

$$
P_{s}^{(\infty)}=A_{\text {in }}^{(\infty)} \frac{e^{-i \omega r}}{r^{1-2 s}}+A_{\text {out }}^{(\infty)} \frac{e^{i \omega r}}{r}
$$

since $\Delta \rightarrow r^{2}$ as $r \rightarrow \infty$.

There are various considerations which must be taken into account in order to obtain results to the required accuracy (at least three significant figures). Firstly, although the horizon boundary condition can not be applied exactly at $r_{H}$ (due to singularities in the boundary condition and the differential equation) the error introduced by applying the condition at $r=r_{c}$ (where $r_{c}-r_{H} \ll r_{H}$ ) must be small. This can be checked by studying changes in the greybody factors for order of magnitude changes in $r_{c}-r_{H}$. Similarly it must be checked that the value of $r$ used as an approximation for 'infinity' does not introduce errors which will affect the accuracy of the result. Care must also be taken that the numerical integration procedure is sufficiently accurate out to large values of $r$ so that significant integration errors are avoided. Finally, for each energy being considered, enough angular momentum modes must be included in the summation so that only higher modes which do not contribute significantly are neglected. For the higher values of $\omega r_{H}$ considered in this work this means that the contributions from in excess of ten angular momentum modes are required.

\section{Numerical Results for Brane Emission}

In this section, we proceed to present our results for the greybody factors and emission rates for brane-localised scalar, fermion and gauge boson fields, as obtained by numerically solving the corresponding equations of motions. The definition of the absorption coefficient $\mathcal{A}_{\ell}$ is different for each type of field due to their different asymptotic behaviour in the far-field regime and also due to more fundamental differences between fields with zero and non-zero spin. We will, therefore, consider each case separately: 


\subsection{Spin 0 fields}

The numerical integration of Eq. (3.3) for $s=0$ yields the solution for the radial function $R_{0}(r)$ which smoothly interpolates between the asymptotic solutions (3.13) and (3.16) in the near-horizon and far-field regimes respectively. The absorption coefficient is easily defined in terms of the in-going and out-going energy fluxes at infinity, or equivalently by the corresponding wave amplitudes in the same asymptotic regime, given by $A_{\text {in }}^{(\infty)}$ and $A_{\text {out }}^{(\infty)}$ respectively. We may thus write Eq. (3.10) in the form $[25]$

$$
\left|\hat{\mathcal{A}}_{\ell}^{(0)}\right|^{2}=1-\left|\hat{\mathcal{R}}_{\ell}^{(0)}\right|^{2}=1-\left|\frac{A_{\text {out }}^{(\infty)}}{A_{\text {in }}^{(\infty)}}\right|^{2},
$$

where $\hat{\mathcal{R}}_{\ell}$ is the corresponding reflection coefficient.

The plot presented in Figure 1 1 shows, for different values of $n$, the greybody factors for the emission of scalar fields on the brane (note that, throughout our numerical analyses, the horizon radius $r_{H}$ is an arbitrary input parameter which remains fixed). The greybody factor is derived by using Eq. (3.8), which is valid for the emission of brane-localised modes, and summing over the angular momentum number $\ell$ (for completeness, the plots include values of $n$ ruled out on astrophysical grounds, i.e. $n=1,2)$. For $n=0$ and $\omega r_{H} \rightarrow 0$, the greybody factor assumes a non-zero value which is equal to $4 \pi r_{H}^{2}$ - that is, the greybody factor for scalar fields with a very low energy is given exactly by the area of the black hole horizon. As the energy increases, the factor soon starts oscillating around the geometrical optics limit $\sigma_{g}=27 \pi r_{H}^{2} / 4$ which corresponds to the spectrum of a black-body with an absorbing area of radius $r_{c}=3 \sqrt{3} r_{H} / 2[31,32]$. If extra dimensions are present, the greybody factor starts from the same asymptotic low-energy value, for any value of

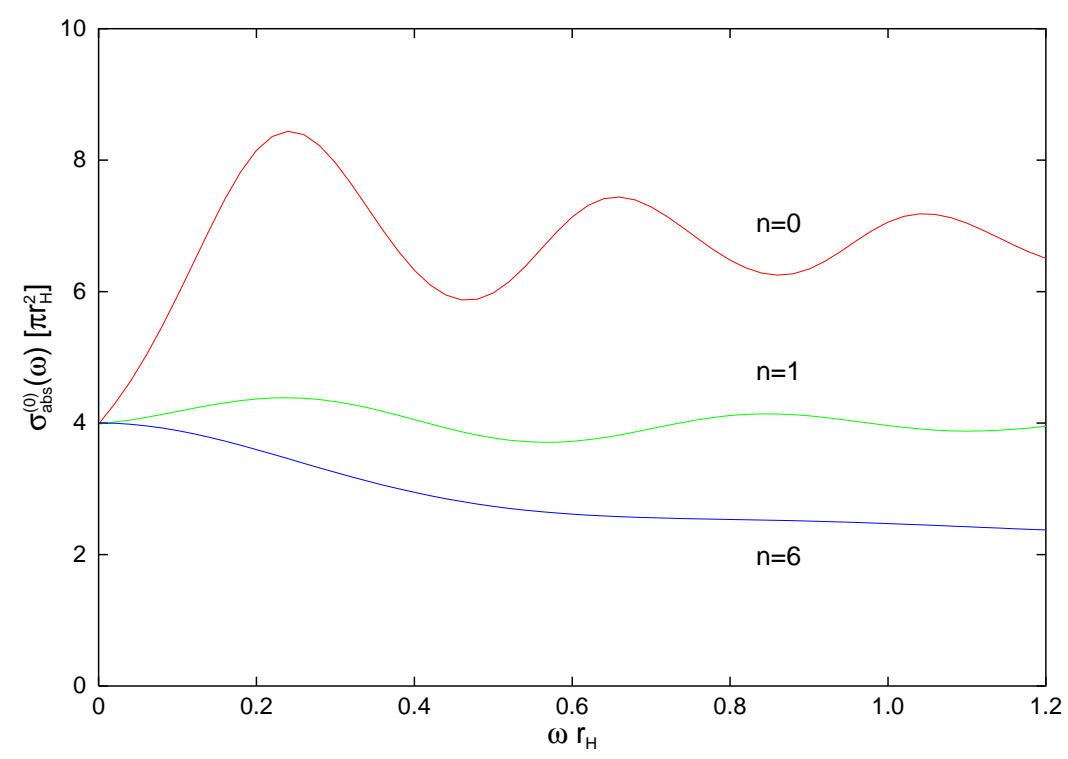

Figure 1: Greybody factors for scalar emission on the brane from a $(4+n) \mathrm{D}$ black hole. 
$n$, and it again starts oscillating around a limiting high-energy value, which is always lower than the 4-dimensional one. This is because the effective radius $r_{c}$ depends on the dimensionality of the bulk spacetime through the metric tensor of the projected spacetime (3.1) in which the particle moves. For arbitrary $n$, it adopts the value [27]

$$
r_{c}=\left(\frac{n+3}{2}\right)^{1 / n+1} \sqrt{\frac{n+3}{n+1}} r_{H} .
$$

The above quantity keeps decreasing as $n$ increases causing the asymptotic greybody factor, $\sigma_{g}=\pi r_{c}^{2}$, to become more and more suppressed as the number of extra dimensions projected onto the brane gets larger.

The power series expression of the greybody factor determined in [25] matches the exact solution only in a very limited low-energy regime. In the limit $\omega r_{H} \rightarrow 0$, the asymptotic value $4 \pi r_{H}^{2}$ is recovered as expected; however, as the energy increases the exact solution rapidly deviates from the behaviour dictated by the dominant term in the $\omega r_{H}$ expansion. This was first demonstrated in [26], where the full analytic result for the greybody factor was determined. The behaviour depicted in Figure 3 of Ref. [26] is much closer to the exact one, shown here in Figure 1, and successfully reproduces the qualitative features including the suppression of the greybody factor as the dimensionality of the bulk spacetime increases. Nevertheless, as previously stated, even that result breaks down in the high-energy regime leaving the exact numerical solution produced here as the only reliable source of information concerning the form of the greybody factor throughout the energy regime.

Proceeding to compute the energy emission rate for scalar fields on the brane, by using Eq. (3.9), we find that the suppression of the greybody factor with $n$ does

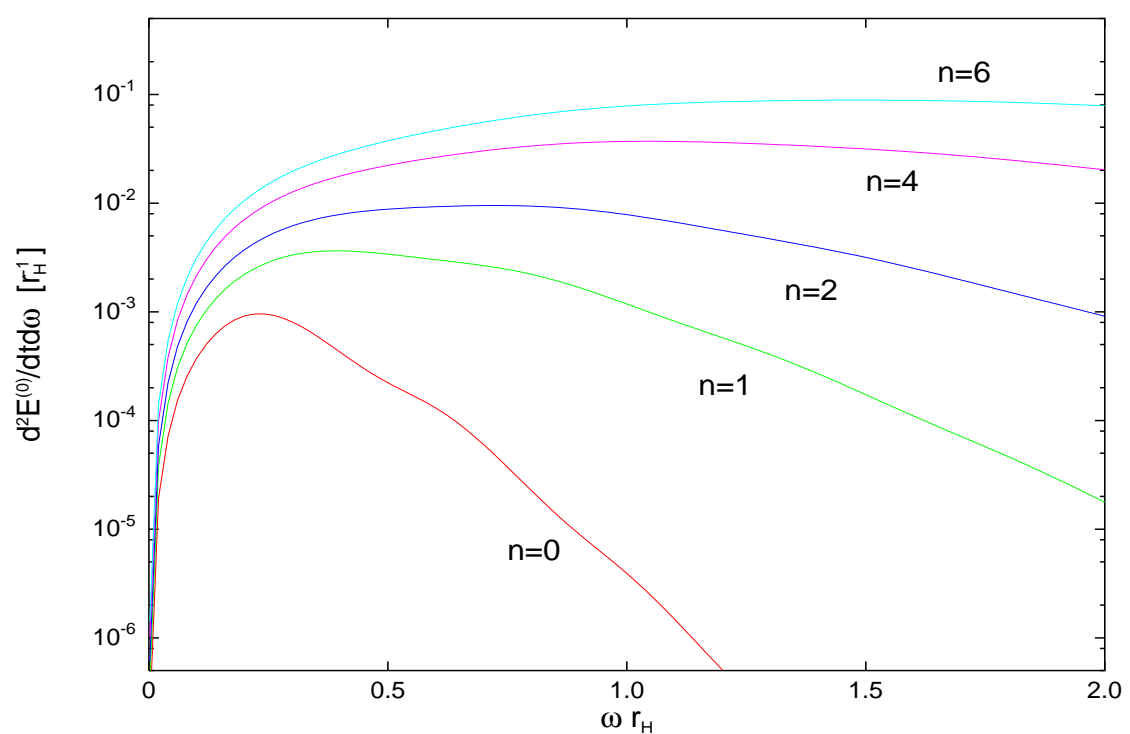

Figure 2: Energy emission rates for scalar fields on the brane from a $(4+n) \mathrm{D}$ black hole. 
not necessarily lead to the suppression of the emission rate itself. The behaviour of the differential energy emission rate in time unit $d t$ and energy interval $d \omega$ is given in Figure 2. The increase in the temperature of the black hole, and thus in its emissivity power, as $n$ increases overcomes the decrease in the value of the greybody factor and leads to a substantial enhancement of the energy emission rate. As it becomes clear from Figure 2, the increase in the number of dimensions projected onto the brane causes the enhancement of the peak value of the emission curve by many orders of magnitude, when compared to the 4-dimensional case. In addition, it leads to a Wien's type of displacement of the peak, i.e. to the shift of the peak towards higher values of the energy parameter $\omega r_{H}$, reflecting the increase in the temperature of the radiating body.

In order to be able to clearly perceive the amount of enhancement of the emission rate of the black hole as the number of extra dimensions projected onto the brane increases, we compute the total flux and power emissivities, for various values of $n$, by integrating Eqs. (2.6) and (2.7) over all energies. The results obtained are displayed in Table 1. The relevant emissivities for different values of $n$ have been normalized in terms of those for $n=0$. From the entries of the table, we may easily see that both the flux of particles produced and the amount of energy radiated per unit time by the black hole on the brane are substantially enhanced, by orders of magnitude, as the number of extra dimensions increases.

\begin{tabular}{|c||c|c|c|c|c|c|c|c|}
\hline \hline & $n=0$ & $n=1$ & $n=2$ & $n=3$ & $n=4$ & $n=5$ & $n=6$ & $n=7$ \\
\hline Flux & 1.0 & 4.75 & 13.0 & 27.4 & 49.3 & 79.9 & 121 & 172 \\
\hline Power & 1.0 & 8.94 & 36.0 & 99.8 & 222 & 429 & 749 & 1220 \\
\hline \hline
\end{tabular}

Table 1 : Flux and Power Emissivities for Scalar Fields on the brane

\subsection{Spin 1/2 fields}

Unlike the case of scalar fields, the study of the emission of fields with non-vanishing spin involves, in principle, the study of fields with more than one component. Equation (3.3) depends on the helicity number $s$ which, upon taking different values, leads to the radial equation for different components of the field. As mentioned in section 3.2, the upper and lower components carry mainly the in-going and out-going parts, respectively, of the field. Although knowledge of both components is necessary in order to construct the complete solution for the emitted field, the determination of either is more than adequate to compute the absorption coefficient $\hat{\mathcal{A}}_{j}$, where $j$ is the total angular momentum number. For example, if the in-going wave is known 
in the case of the emission of fields with spin $s=1 / 2$, Eq. (3.10) may be directly writen as [26] [38]

$$
\left|\hat{\mathcal{A}}_{j}^{(1 / 2)}\right|^{2}=\left|\frac{A_{i n}^{(h)}}{A_{i n}^{(\infty)}}\right|^{2} .
$$

The above follows by defining the incoming flux of a fermionic field as the radial component of the conserved current, $J^{\mu}=\sqrt{2} \sigma_{A B}^{\mu} \Psi^{A} \bar{\Psi}^{B}$, integrated over a twodimensional sphere and evaluated both at the horizon and infinity.

The greybody factor is again related to the aforementioned absorption probability through Eq. (3.8) with $\ell$ being replaced by $j$. By numerically solving the radial equation Eq. (3.3) and computing $\hat{\mathcal{A}}_{j}^{(1 / 2)}$, we obtain the behaviour of the greybody factor, in terms of the energy parameter $\omega r_{H}$ and number of extra dimensions $n$, depicted in Figure 3. At low energies the greybody factor assumes, as in the case of scalar fields, a non-zero asymptotic value; this depends on the dimensionality of spacetime and increases with increasing $n$. The enhancement of $\sigma_{\text {abs }}^{(1 / 2)}(\omega)$ with $n$ in the low-energy regime persists up to intermediate values of $\omega r_{H}$, after which the situation is reversed: as $n$ takes on larger values, the greybody factor becomes more and more suppressed. The complete analytic results derived in [26] successfully describe both the low-energy behaviour and the existence of the 'turning point'; however, as expected, they fail to give accurate information for the high-energy regime. Figure 3 shows that at high energies the greybody factors for fermion fields oscillate around the same asymptotic values [determined by the effective radius (4.2)] as for scalar fields.

The energy emission rate for fermion fields on the brane, for various values of $n$, is shown in Figure 4 . As $n$ increases, it is found to be significantly enhanced, both

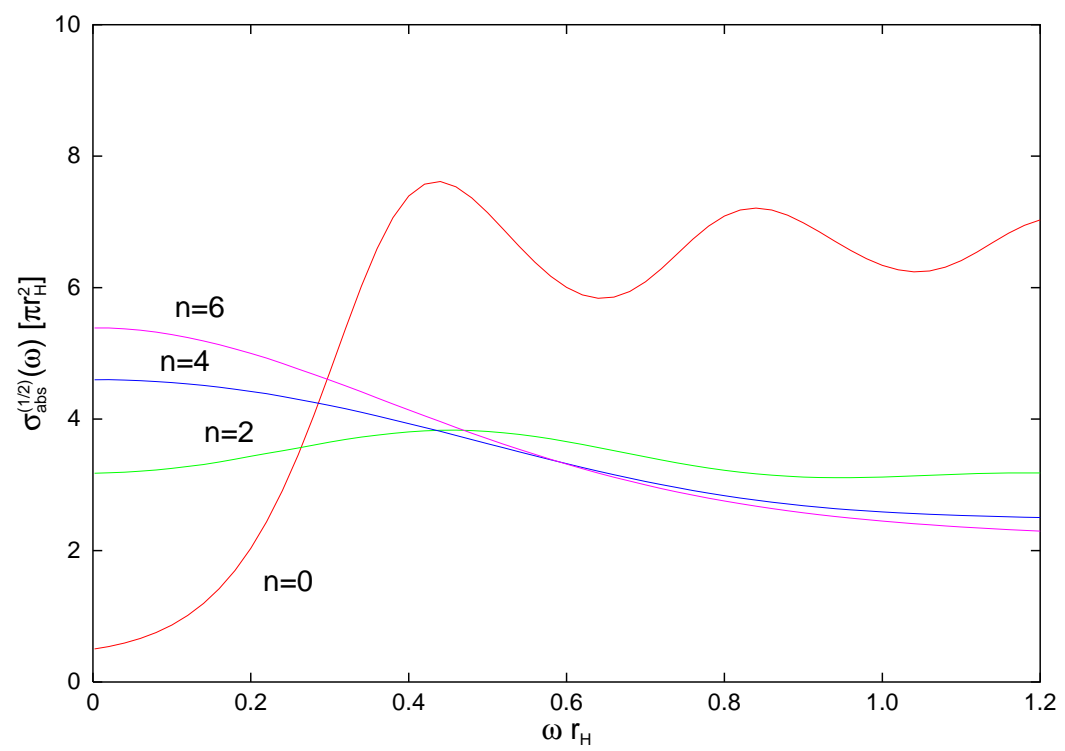

Figure 3: Greybody factors for fermion emission on the brane from a $(4+n) \mathrm{D}$ black hole. 


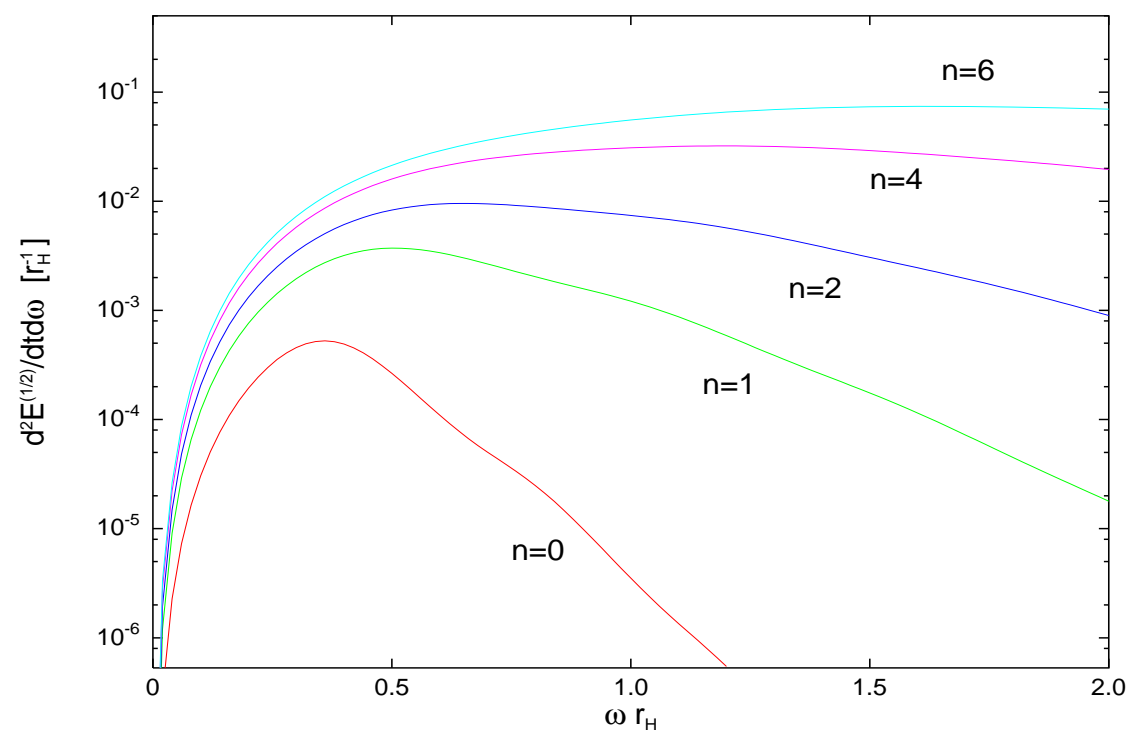

Figure 4: Energy emission rates for fermions on the brane from a $(4+n) \mathrm{D}$ black hole.

at low and high energies, mainly due to the increase in the temperature of the black hole. The emission curves exhibit the same features as for the emission of scalar fields, i.e. increase of the height of the peak by orders of magnitude and shift of the peak towards higher energies. Some quantitative results regarding the enhancement of both the flux and power spectra for the emission of fermions, as $n$ increases, are given in Table 2. Once again, the enhancement in both spectra with $n$ is indeed substantial, and even more important compared to the one for scalar emission.

\begin{tabular}{|c||c|c|c|c|c|c|c|c|}
\hline \hline & $n=0$ & $n=1$ & $n=2$ & $n=3$ & $n=4$ & $n=5$ & $n=6$ & $n=7$ \\
\hline Flux & 1.0 & 9.05 & 27.6 & 58.2 & 103 & 163 & 240 & 335 \\
\hline Power & 1.0 & 14.2 & 59.5 & 162 & 352 & 664 & 1140 & 1830 \\
\hline \hline
\end{tabular}

Table 2 : Flux and Power Emissivities for Fermions on the brane

\subsection{Spin 1 fields}

In the case of the emission of gauge boson fields, the incoming flux can be computed by the $(t r)$-component of the energy-momentum tensor, $T^{\mu \nu}=2 \sigma_{A A^{\prime}}^{\mu} \sigma_{B B^{\prime}}^{\nu} \Psi^{A B} \bar{\Psi}^{A^{\prime} B^{\prime}}$, integrated again over a two-dimensional sphere and evaluated at the horizon and infinity. By making use of the solution for the in-going wave, the following expression for the absorption probability, Eq. (3.10), is obtained [26] [38]:

$$
\left|\hat{\mathcal{A}}_{j}^{(1)}\right|^{2}=\frac{1}{r_{H}^{2}}\left|\frac{A_{i n}^{(h)}}{A_{i n}^{(\infty)}}\right|^{2} .
$$




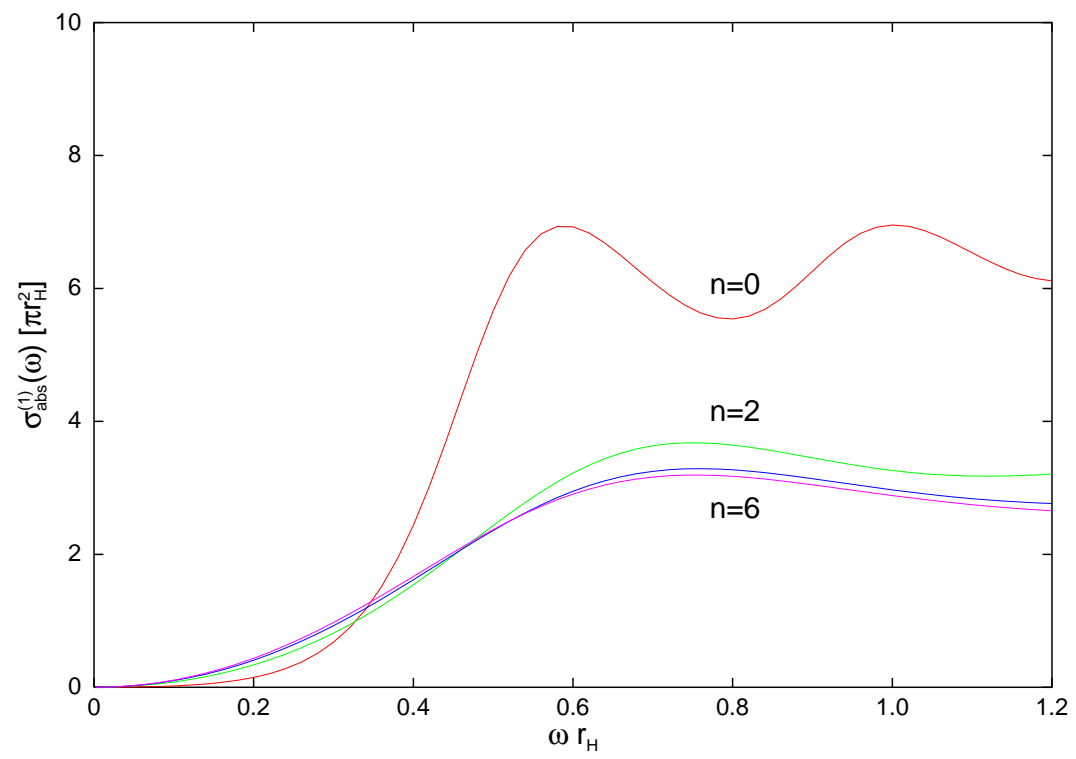

Figure 5: Greybody factors for gauge boson emission on the brane from a $(4+n)$ D black hole.

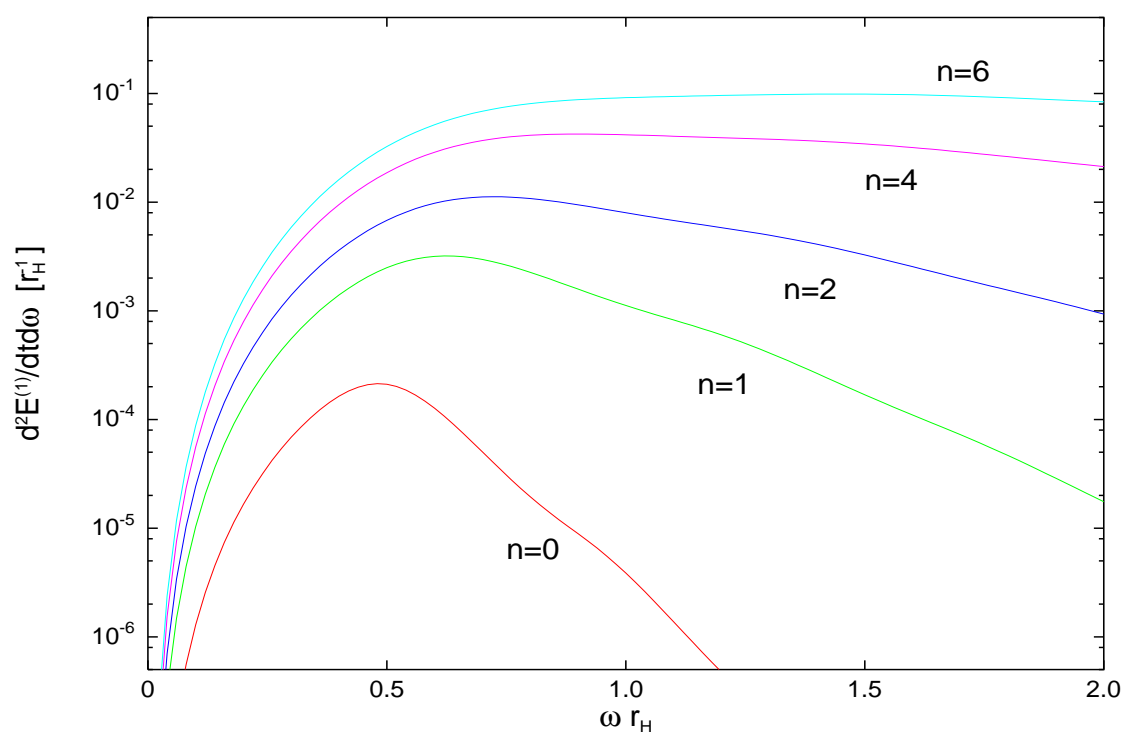

Figure 6: Energy emission rates for gauge fields on the brane from a $(4+n) \mathrm{D}$ black hole.

The exact results for the greybody factors and energy emission rates for gauge boson fields are given in Figures 5 and 6 respectively. A distinct feature of the greybody factor for gauge fields, already known from the 4-dimensional case, is that it vanishes when $\omega r_{H} \rightarrow 0$. The same behaviour is observed for every value of the number of extra dimensions. This result leads to the suppression of the energy emission rate, in the low-energy regime, compared to the ones for scalar and fermion fields. Up to intermediate energies the greybody factors exhibit the same enhancement with increasing $n$ as in the case of fermion fields, and as it was analytically shown in [26]. 
A similar asymptotic behaviour, as in the previous cases, is observed in the highenergy regime with each greybody factor assuming, after oscillation, the geometrical optics value which decreases with increasing $n$. This result establishes the existence of a universal behaviour of all types of particles emitted by the black hole at high energies. This behaviour is independent of the particle spin but strongly dependent on the number of extra dimensions projected onto the brane.

We may finally obtain, as in the previous cases, the total flux and power emissivities for the emission of gauge fields on the brane, in terms of the number of extra dimensions $n$. The exact results obtained by numerically integrating over all energies are given in Table 3. As anticipated, the same pattern of enhancement with $n$ is also observed for the emission of gauge bosons. It is worth noting that the enhancement observed in this case is the largest amongst all types of particles - this result points to the dominance of the emission of gauge bosons over other types of particles in models with large values of $n$, as we will shortly see.

\begin{tabular}{|c||c|c|c|c|c|c|c|c|}
\hline \hline & $n=0$ & $n=1$ & $n=2$ & $n=3$ & $n=4$ & $n=5$ & $n=6$ & $n=7$ \\
\hline Flux & 1.0 & 19.2 & 80.6 & 204 & 403 & 689 & 1070 & 1560 \\
\hline Power & 1.0 & 27.1 & 144 & 441 & 1020 & 2000 & 3530 & 5740 \\
\hline \hline
\end{tabular}

Table 3 : Flux and Power Emissivities for Gauge Fields on the brane

\subsection{Relative Emissivities for different species}

It would be interesting to investigate how the relative numbers of scalars, fermions and gauge bosons, emitted by the black hole on the brane, change as the number of extra dimensions projected onto the brane varies. In other words, we would like to know what type of particles the black hole prefers to emit, for different values of $n$, and what part of the total energy each particular type of particle carries away during emission.

Comparing the energy emission rates (computed in the previous sub-sections), for different types of particles and for fixed $n$, can give us the qualitative behaviour. Figures 7(a) and 7(b) show, with a linear scale, the above quantities for $n=0$ and $n=6$ respectively - note that these two figures summarize very clearly the effects discussed in the previous sub-sections, i.e. the orders-of-magnitude enhancement of the emission rates and the Wien's displacement of the peak to the right, as $n$ increases. Figure Ø(a) reveals that, in the absence of any extra dimensions, most of the energy of the black hole emitted on the brane is in the form of scalar particles; the next most important are the fermion fields, and less significant are the gauge 

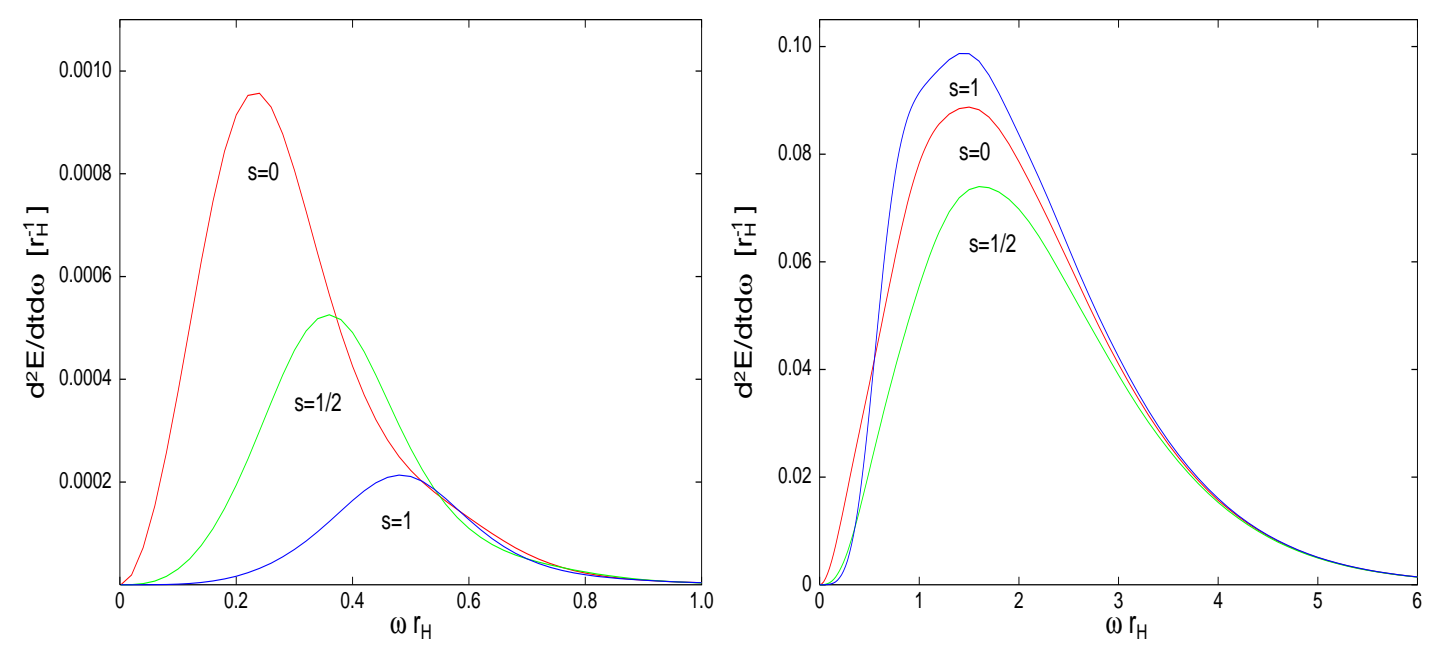

Figure 7: (a): Energy emission rates for the emission of scalars, fermions and gauge bosons on the brane for $n=0$. (b) : The same, but for $n=6$.

bosons. As $n$ increases, the emission rates for all species are enhanced but not at the same rate. Figure 7 (b) clearly shows that, for a large number of extra dimensions, the most effective 'channel' during the emission of brane localized modes is that of gauge bosons; the scalar and fermion fields follow second and third respectively. The change in the flux spectra, i.e. in the number of particles produced by the black hole on the brane, for each species is similar as $n$ increases.

In order to quantify the above behaviour, we computed the relative emissivities for scalars, fermions and gauge bosons emitted on the brane by integrating the flux (2.6) and energy (2.7) emission spectra, for different types of particles, over all energies. The relative emissivities obtained in this way are shown in Tables 1 and 5 (they are normalized to the scalar values). Note that the ratios for $n \geq 1$ are available for the first time in the literature as a result of this numerical work, while the $n=0$

\begin{tabular}{|c||c|c|c|}
\hline \hline & $s=0$ & $s=\frac{1}{2}$ & $s=1$ \\
\hline$n=0$ & 1.0 & 0.37 & 0.11 \\
$n=1$ & 1.0 & 0.70 & 0.45 \\
$n=2$ & 1.0 & 0.77 & 0.69 \\
$n=3$ & 1.0 & 0.78 & 0.83 \\
$n=4$ & 1.0 & 0.76 & 0.91 \\
$n=5$ & 1.0 & 0.74 & 0.96 \\
$n=6$ & 1.0 & 0.73 & 0.99 \\
$n=7$ & 1.0 & 0.71 & 1.01 \\
\hline Blackbody & 1.0 & 0.75 & 1.0 \\
\hline \hline
\end{tabular}

Table 4: Flux emission ratios

\begin{tabular}{|c||c|c|c|}
\hline \hline & $s=0$ & $s=\frac{1}{2}$ & $s=1$ \\
\hline$n=0$ & 1.0 & 0.55 & 0.23 \\
$n=1$ & 1.0 & 0.87 & 0.69 \\
$n=2$ & 1.0 & 0.91 & 0.91 \\
$n=3$ & 1.0 & 0.89 & 1.00 \\
$n=4$ & 1.0 & 0.87 & 1.04 \\
$n=5$ & 1.0 & 0.85 & 1.06 \\
$n=6$ & 1.0 & 0.84 & 1.06 \\
$n=7$ & 1.0 & 0.82 & 1.07 \\
\hline Blackbody & 1.0 & 0.87 & 1.0 \\
\hline \hline
\end{tabular}

Table 5: Power emission ratios 
results would appear to be the most accurate ones available. The entries in these tables reflect the qualitative behaviour discussed above for some extreme values of the number of extra dimensions. For $n=0$, the scalar fields are indeed the type of particles which are most commonly produced and the ones which carry away most of the energy of the black hole emitted on the brane; the fermion and gauge fields carry approximately $1 / 2$ and $1 / 4$, respectively, of the energy emitted in the form of the scalar fields, and their fluxes are only $1 / 3$ and $1 / 10$ of the scalar flux. For intermediate values of $n$, the fermion and gauge boson emissivities have been considerably enhanced compared to the scalar one and have become of approximately the same magnitude - e.g. for $n=2$ the amount of energy spent by the black hole for the emission of fermions and gauge bosons is exactly the same, although the net number of gauge bosons is still sub-dominant. For large values of $n$, the situation is reversed: the gauge bosons dominate both flux and power spectra, with the emission of fermions being the least effective 'channel' both in terms of number of particles produced and energy emitted. We remind the reader that the above results refer to the emission of individual scalar, fermionic or bosonic degrees of freedom and not to elementary particles.

We may thus conclude that not only the magnitude but also the type of flux and power spectra produced by a small, higher-dimensional black hole strongly depends on the number of extra dimensions projected onto the brane. Therefore, upon detecting the Hawking radiation from such objects, the above distinctive feature could serve as an alternative way to determine the number of extra dimensions that exist in nature.

\section{Emission in the Bulk}

An extremely important question regarding the emission of particles by a higherdimensional black hole is how much of this energy is radiated onto the brane and how much is lost in the bulk. In the former case, the emitted particles are zero-mode gravitons and scalar fields as well as Standard Model fermions and gauge bosons, while in the latter case all emitted energy is in the form of massive Kaluza-Klein gravitons and, possibly, scalar fields. In [27], it was shown that the whole tower of KK excitations of a given particle carries approximately the same amount of energy as the massless zero-mode particle emitted on the brane. Combining this result with the fact that many more types of particles live on the brane than in the bulk, it was concluded that most of the energy of the black hole goes into brane modes. The results obtained in [27] were only approximate since the dependence of the greybody factor on the energy of the emitted particle was ignored and the (low-energy valid) geometric expression for the area of the horizon was used instead.

In order to provide an accurate answer to the question of how much energy is emitted into the bulk compared to on the brane, it is imperative that the dependence 
of the greybody factor on both the energy and number of extra dimensions is taken into account (for a similar but incomplete - since the dependence on the energy was again ignored - argument in this direction, see [41]). In this section, we first thoroughly investigate the emission of scalar modes in the bulk and produce exact numerical results for the behaviour of greybody factors and energy emission rates in terms of the energy and number of extra dimensions. Subsequently, we address the above question and provide a definite answer by computing, for different values of $n$, the total bulk-to-brane relative emissivities.

\subsection{Greybody Factors and Emission Rates}

In this section, we turn to the investigation of the emission of bulk modes from a higher-dimensional black hole. This analysis is relevant for gravitons and scalar fields, and requires knowledge of the solutions of the corresponding equations of motion in the bulk. Here, we will focus on the case of scalar fields for which the bulk equation is known - the emission of bulk scalar modes was previously studied analytically, in the low-energy regime, in [25].

A scalar field propagating in the background of a higher-dimensional, nonrotating, Schwarzschild-like black hole, whose line-element is given by Eq. (2.1), satisfies the following equation of motion [25]

$$
\frac{h(r)}{r^{n+2}} \frac{d}{d r}\left[h(r) r^{n+2} \frac{d R}{d r}\right]+\left[\omega^{2}-\frac{h(r)}{r^{2}} \ell(\ell+n+1)\right] R=0 .
$$

As in the case of the emission of particles on the brane, the determination of the greybody factor for emission in the bulk demands solving the above equation over the whole radial domain. The exact solution for the radial function must interpolate between the near-horizon and far-field asymptotic solutions, given by

$$
R^{(h)}=A_{\text {in }}^{(h)} e^{-i \omega r^{*}}+A_{o u t}^{(h)} e^{i \omega r^{*}}
$$

and

$$
R^{(\infty)}=A_{\text {in }}^{(\infty)} \frac{e^{-i \omega r}}{\sqrt{r^{n+2}}}+A_{\text {out }}^{(\infty)} \frac{e^{i \omega r}}{\sqrt{r^{n+2}}},
$$

respectively. We impose again the boundary condition that no out-going solution should exist near the horizon of the black hole, and therefore we set $A_{\text {out }}^{(h)}=0$. The solution at infinity comprises, as usual, both in-going and out-going modes.

The expression for the absorption probability $\left|\tilde{\mathcal{A}}_{\ell}\right|^{2}$ may then be derived either by using Eq. (4.1) or by calculating directly the ratio $\left|A_{i n}^{(h)} / A_{i n}^{(\infty)}\right|$ - note that, henceforth, quantities with a tilde denote bulk quantities (as opposed to brane quantities which carry a hat). The corresponding greybody factor $\tilde{\sigma}_{\ell}(\omega)$ may then be determined by using the relation (2.8). The dimensionality of the greybody factor changes as the number of extra dimensions $n$ varies; therefore, in order to be able 
to compare its values for different $n$, we normalize its expression to the area of the horizon of the $(4+n)$-dimensional black hole. Thus, we rewrite Eq. (2.8) in the form

$$
\tilde{\sigma}_{\ell}(\omega)=\frac{2^{n}}{\pi} \Gamma\left[\frac{n+3}{2}\right]^{2} \frac{\tilde{A}_{H}}{\left(\omega r_{H}\right)^{n+2}} \tilde{N}_{\ell}\left|\tilde{\mathcal{A}}_{\ell}\right|^{2},
$$

where $\tilde{N}_{\ell}$ is the multiplicity of states corresponding to the same partial wave $\ell$, given for a $(4+n)$-dimensional spacetime by

$$
\tilde{N}_{\ell}=\frac{(2 \ell+n+1)(\ell+n) !}{\ell !(n+1) !},
$$

and $\tilde{A}_{H}$ is the horizon area in the bulk defined as

$$
\begin{aligned}
\tilde{A}_{H} & =r_{H}^{n+2} \int_{0}^{2 \pi} d \varphi \prod_{k=1}^{n+1} \int_{0}^{\pi} \sin ^{k} \theta_{n+1} d \sin \theta_{n+1} \\
& =r_{H}^{n+2}(2 \pi) \prod_{k=1}^{n+1} \sqrt{\pi} \frac{\Gamma[(k+1) / 2]}{\Gamma[(k+2) / 2]} \\
& =r_{H}^{n+2}(2 \pi) \pi^{(n+1) / 2} \Gamma\left[\frac{n+3}{2}\right]^{-1}
\end{aligned}
$$

Equation (5.4) allows us to compute the low-energy limit of the greybody factor once the corresponding expression of the absorption coefficient is determined. Analytic results for $\tilde{\mathcal{A}}_{\ell}$ were derived in [25] by solving Eq. (5.1) in the two asymptotic regimes, the near-horizon and far-field, and matching them in an intermediate zone. It was found that the low-energy expression of the absorption coefficient, for $\ell=0$, has the form

$$
\left|\tilde{\mathcal{A}}_{0}\right|^{2}=\left(\frac{\omega r_{H}}{2}\right)^{n+2} \frac{4 \pi}{\Gamma[(n+3) / 2]^{2}}+\ldots,
$$

where the dots denote higher-order terms in the power-expansion in $\omega r_{H}$. These terms, as well as the corresponding expressions of $\tilde{\mathcal{A}}_{\ell}$ for higher partial waves, vanish quickly in the limit $\omega r_{H} \rightarrow 0$, leaving the above term as the dominant one. Substituting into Eq. (5.4), we easily see that, in the low-energy regime, the greybody factor is given by the area $\tilde{A}_{H}$ of the horizon of the black hole. This behaviour is similar to the 4-dimensional case; however, note that, in this case, the area of the horizon changes as $n$ varies.

In the high-energy regime, we anticipate recovering the equivalent of the $(4+n)$ dimensional geometrical optics limit. In four dimensions, the low-energy limit for the greybody factor, $4 \pi r_{H}^{2}$, goes over to the geometrical optics value $\pi r_{c}^{2}$ at high energy. This has led to the naïve generalization that, in an arbitrary number of dimensions, the high-energy expression for the greybody factor will be approximately $\Omega_{n+2} r_{c}^{n+2} / 4$, where $\Omega_{n+2}$ is the volume element of the $(n+2)$-dimensional sphere. We will shortly see that this is in fact an over-estimate of the value of the greybody 
factor in the high-energy regime. As in four dimensions, we will assume that, for large values of the energy of the scattered particle, the greybody factor becomes equal to the area of an absorptive body of radius $r_{c}$ which is projected on a plane parallel to the one of the orbit of the moving particle [31]. According to Ref. [27], the value of the effective radius $r_{c}$ remains the same both for bulk and brane particles and it is given by Eq. (4.2). The area of the absorptive body depends strongly on the dimensionality of spacetime and its calculation demands setting one of the azimuthal angles equal to $\pi / 2$. A careful calculation reveals that the 'projected' area is given by

$$
\tilde{A}_{p}=\frac{2 \pi}{(n+2)} \frac{\pi^{n / 2}}{\Gamma[(n+2) / 2]} r_{c}^{n+2}=\frac{1}{n+2} \Omega_{n+1} r_{c}^{n+2} .
$$

The above relation reduces to the usual 4-dimensional result $\tilde{A}_{p}=\pi r_{c}^{2}$, for $n=$ 0 , while it leads to values reduced by $50 \%$, compared to the naïve expression $\Omega_{n+2} r_{c}^{n+2} / 4$, for higher values of $n$. Assuming that the greybody factor at high energies becomes equal to the absorptive area $\tilde{A}_{p}$ of radius $r_{c}$, we may explicitly write:

$$
\begin{aligned}
\tilde{\sigma}_{\ell}(\omega) & =\frac{1}{n+2} \frac{\Omega_{n+1}}{\Omega_{n+2}}\left(\frac{r_{c}}{r_{H}}\right)^{n+2} \tilde{A}_{H} \\
& =\frac{1}{\sqrt{\pi}(n+2)} \frac{\Gamma[(n+3) / 2]}{\Gamma[(n+2) / 2]}\left(\frac{n+3}{2}\right)^{(n+2) /(n+1)}\left(\frac{n+3}{n+1}\right)^{(n+2) / 2} \tilde{A}_{H} .
\end{aligned}
$$

In the above, we have used the same normalization, in terms of the area of the $(4+n)$-dimensional horizon, as in the low-energy regime.

Turning now to the numerical analysis, we may find the expressions of the greybody factors, for various values of $n$ and for all energy regimes, by using Eq. (5.4) and the exact numerical results for the absorption coefficients. Their behaviour is shown in Figure 8. As it was anticipated after the above discussion, the normalized greybody factors, in the low-energy regime, tend to unity for all values of $n$, as each one of them adopts the value of the area of the black hole horizon to which it has been normalized. As in the case of the emission of scalar fields on the brane, the greybody factors are suppressed in the low-energy regime, as $n$ increases, while they start oscillating at intermediate energies and finally adopt their asymptotic high-energy limit. A simple numerical analysis shows that the naïve expression $\Omega_{n+2} r_{c}^{n+2} / 4$ fails to describe the high-energy asymptotic limits for all values of $n$ larger than zero. On the other hand, the expression (5.9) gives asymptotic values which are much closer to the ones depicted in Figure 8, but these values still deviate from the exact ones as $n$ increases. A more sophisticated analysis is thus necessary in order to determine the exact high-energy limit for emission in the bulk which might lead either to the reconsideration of the absorptive-area argument for $n \geq 1$, or to the introduction of a correcting term that suppresses the asymptotic value (5.9) as $n$ increases. 


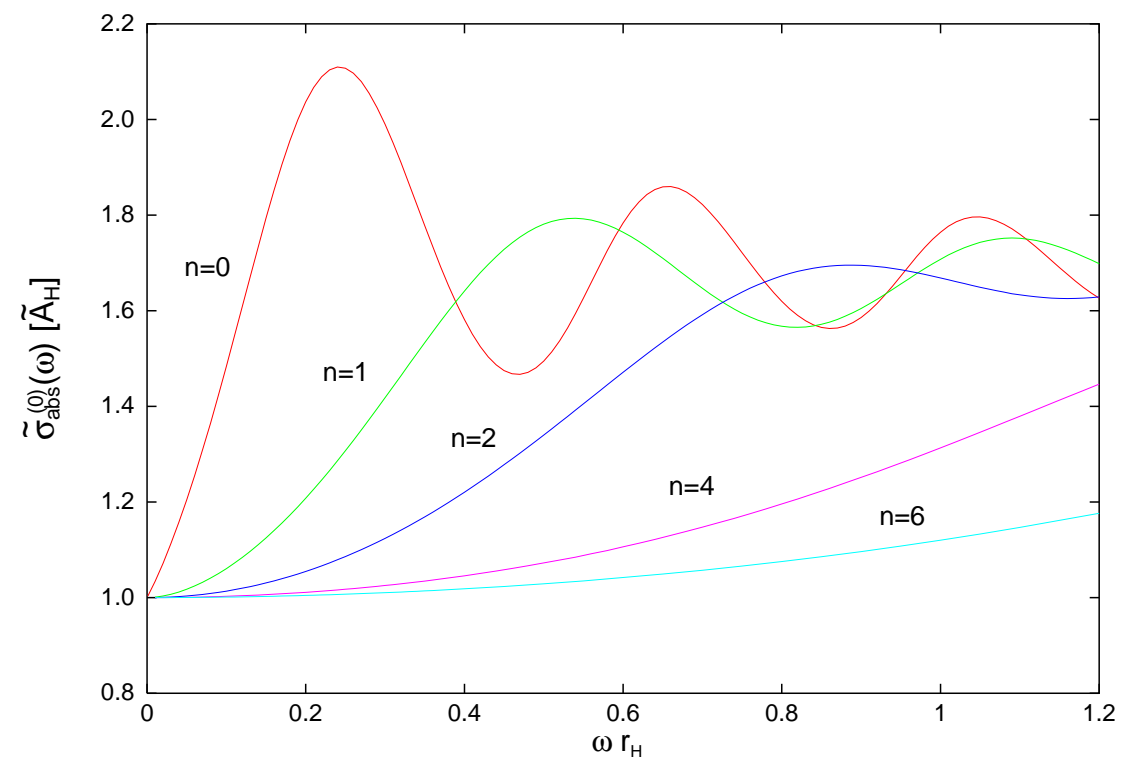

Figure 8: Greybody factors for scalar emission in the bulk from a $(4+n) \mathrm{D}$ black hole.

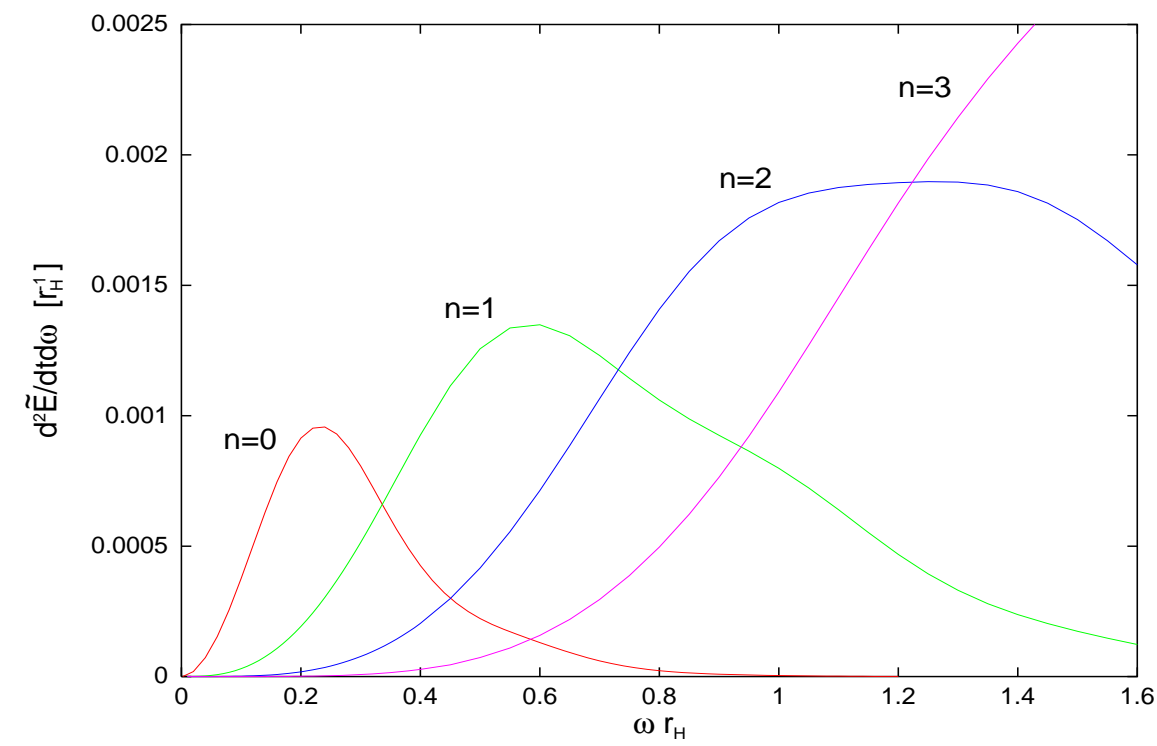

Figure 9: Energy emission rates for scalar fields in the bulk from a $(4+n)$ D black hole.

In general, the suppression of the greybody factor for bulk emission at low energies is milder than the one for brane emission. This, however, does not lead to higher emission rates for the bulk modes compared to those for brane modes: the integration over the phase-space in Eq. (2.7) involves powers of $\left(\omega r_{H}\right)$ which cause an increasingly suppressive effect, in the low-energy regime, as $n$ increases. Nevertheless, the increase in the temperature of the black hole, which is again given by $T_{B H}=(n+1) / 4 \pi r_{H}$, eventually overcomes the decrease in the value of the greybody factor and causes the enhancement of the emission rate with $n$ at high energies. 
The behaviour of the differential energy emission rates as a function of the energy parameter $\omega r_{H}$ and for some indicative numbers of extra dimensions is depicted in Figure 9. After the aforementioned suppression at the low-energy regime as $n$ increases, the energy emission rates soon become enhanced, with the peak of the curve becoming higher and corresponding to larger values of $\omega r_{H}$. It is worth noting that the full analytic results, which may be derived from the analysis of Ref. [25], successfully describe the low-energy behaviour of both the greybody factors and the energy emission rates for the emission of scalar fields in the bulk.

\subsection{Bulk-to-Brane Relative Emissivities}

In this section, we perform an analysis aiming at providing an answer to the question of the relative bulk-to-brane emissivity. We evaluate the differential energy emission rates in the bulk and on the brane, and compare the two quantities for different numbers of extra dimensions.

Equation (2.7) for energy emission in the bulk may be alternatively written, in terms of the absorption coefficient, as

$$
\frac{d \tilde{E}(\omega)}{d t}=\sum_{\ell} \tilde{N}_{\ell}\left|\tilde{\mathcal{A}}_{\ell}\right|^{2} \frac{\omega}{\exp \left(\omega / T_{B H}\right)-1} \frac{d \omega}{2 \pi} .
$$

The above must be compared with the corresponding expression for the emission of brane-localized modes given by

$$
\frac{d \hat{E}(\omega)}{d t}=\sum_{\ell} \hat{N}_{\ell}\left|\hat{\mathcal{A}}_{\ell}\right|^{2} \frac{\omega}{\exp \left(\omega / T_{B H}\right)-1} \frac{d \omega}{2 \pi},
$$

where $\hat{N}_{\ell}=2 \ell+1$. Since both bulk and brane modes 'feel' the same temperature, the relative bulk-to-brane ratio of the two energy emission rates will be simply given by the expression

$$
\frac{d \tilde{E} / d t}{d \hat{E} / d t}=\frac{\sum_{\ell} \tilde{N}_{\ell}\left|\tilde{\mathcal{A}}_{\ell}\right|^{2}}{\sum_{\ell} \hat{N}_{\ell}\left|\hat{\mathcal{A}}_{\ell}\right|^{2}}
$$

and will depend on the scaling of the multiplicities of states and the absorption coefficients ${ }^{2}$ with $n$. As is clear from Eq. (5.5), the multiplicity of bulk modes $\tilde{N}_{\ell}$ increases quickly for increasing $n$, while $\hat{N}_{\ell}$ remains the same. However, it turns out that the enhancement of the absorption probability $\left|\hat{\mathcal{A}}_{\ell}\right|^{2}$ for brane emission, as $n$ increases, is in total considerably greater than the one for bulk emission. This leads to the dominance of the emission of brane-localized modes over bulk modes, which as we will see becomes stronger for intermediate values of $n$. The behaviour of this ratio is depicted in Figure 10. We observe that, in the low-energy regime, the

\footnotetext{
${ }^{2}$ Note that the absorption coefficients related to the greybody factors through Eq. (2.8), with a multiplicative coefficient that depends both on $\omega r_{H}$ and $n$, might have a completely different behaviour from the greybody factors themselves.
} 


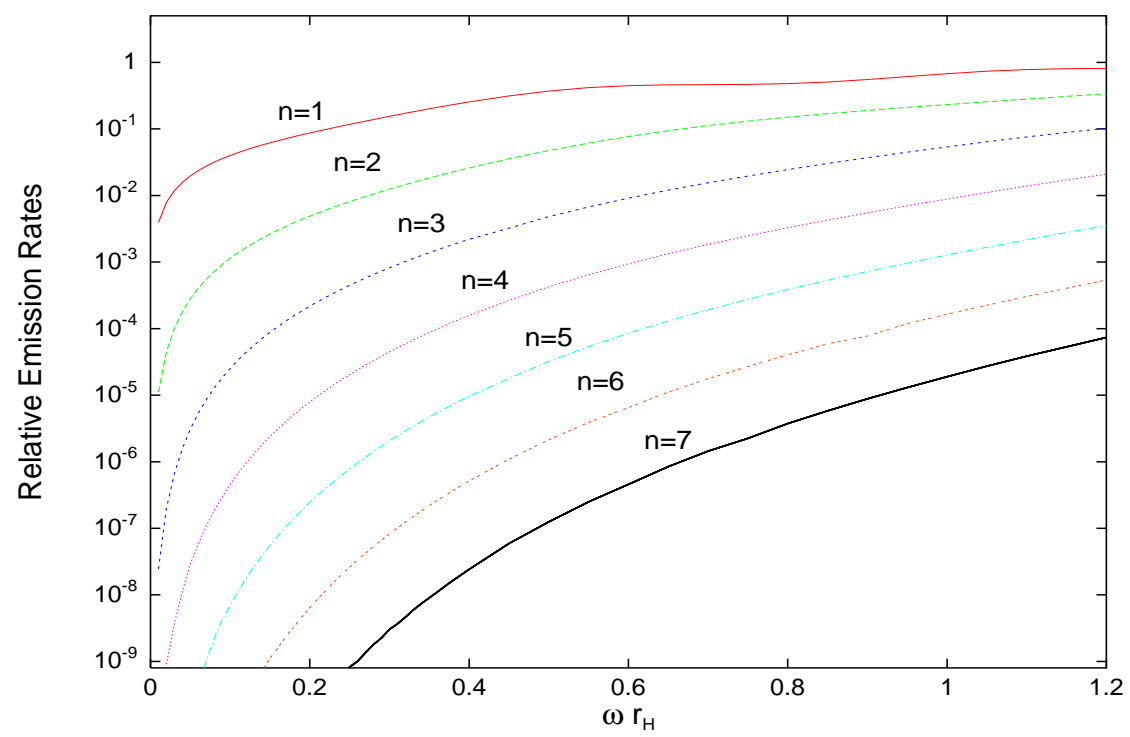

Figure 10: Bulk-to-Brane energy emission rates for scalar fields from a $(4+n) \mathrm{D}$ black hole.

ratio is suppressed, for large values of $n$, by many orders of magnitude, compared to the value of unity for $n=0$. In the high-energy regime on the other hand, the suppression becomes smaller and the ratio seems to approach unity. A more careful examination reveals that, in fact, the bulk modes dominate over the brane modes in a limited high-energy regime that becomes broader as $n$ increases.

A definite conclusion regarding the relative amount of energy which is emitted in the two 'channels' - bulk and brane - can only be drawn if the corresponding total energy emissivities can be computed. By integrating the areas under the bulk and brane energy emission rate curves, for fixed value of $n$, and taking their ratio we were able to determine the relative energy emission rates. The results obtained, for values of $n$ from 1 to 7 , are given in Table 6 .

\begin{tabular}{|c||c|c|c|c|c|c|c|c|}
\hline \hline & $n=0$ & $n=1$ & $n=2$ & $n=3$ & $n=4$ & $n=5$ & $n=6$ & $n=7$ \\
\hline Bulk/Brane & 1.0 & 0.40 & 0.24 & 0.22 & 0.24 & 0.33 & 0.52 & 0.93 \\
\hline \hline
\end{tabular}

Table 6: Relative Bulk-to-Brane Energy Emission Rates for Scalar Fields

From the entries of the above Table, it becomes clear that the emission of branelocalized scalar modes is indeed dominant, in terms of the energy emitted, for all values of $n$ greater than zero and up to 7 . As $n$ increases, the ratio of bulk to brane emission gradually becomes smaller, and becomes particularly suppressed for intermediate values of the number of extra dimensions, i.e. $n=2,3,4$ and 5 ; in these cases, the total energy emitted in the bulk varies between $1 / 3$ and $1 / 4$ approximately 
of that emitted on the brane. As $n$ increases further, the high-energy dominance of the bulk modes, mentioned above, gives a boost to the value of the bulk-to-brane ratio thus causing its increase - nevertheless, the energy ratio never exceeds unity. This means that most of the energy of the higher-dimensional black hole, in the 'scalar' channel, is emitted directly on the brane, in the form of zero-mode scalar fields instead of modes in the bulk.

The above analysis provides exact, accurate results for the energy emission rates for brane and bulk scalar modes and gives considerable support to earlier, more heuristic, arguments [27], according to which a $(4+n)$-dimensional black hole emits mainly brane modes. A complete confirmation demands performing a similar analysis for the emission of gravitons and the results will be reported elsewhere.

\section{Conclusions}

The revival of the idea of the existence of extra space-like dimensions in nature has led to the formulation of theories that allow for a significantly lower energy scale at which gravity becomes strong. This has opened the way for the proposal of the creation of miniature higher-dimensional black holes during collisions of energetic particles in the earth's atmosphere or at ground-based particle colliders. These black holes have a temperature that depends on details of the higher-dimensional background, such as the horizon radius and the number of spatial dimensions, and, upon implementation of quantum effects, emit Hawking radiation. In this paper, we have studied in an exact way the emission of Hawking radiation both on the brane and in the bulk from non-rotating, uncharged $(4+n)$-dimensional black holes, and searched for distinctive features in the radiation spectra which would allow us to determine the number of extra dimensions that exist in nature. At the same time, we provided answers to questions that had remained open from previous analyses in the literature.

Focusing initially on the emission of brane-localized modes, we have derived a master equation describing the motion of a field with arbitrary spin $s$ in the spherically-symmetric black hole background induced on the brane. We then provided exact, numerical results for the greybody factors and emission rates for scalars, fermions and gauge bosons. These results are valid in all energy regimes, and agree with the full analytic results derived in [26] at low- and intermediate-energy scales. The greybody factors interpolate between the low- and high-energy asymptotic limits in a way that depends both on their spin and the dimensionality of spacetime: while they adopt the same asymptotic values (which decrease as $n$ increases) for all particle species in the high-energy regime, in the low-energy limit the greybody factors are enhanced as $n$ increases for $s=1$ and $\frac{1}{2}$, and suppressed for $s=0$. Therefore, their implementation in the calculation of the emission rates is imperative if one wants to derive accurate results for these quantities. By doing that, the energy emission rates obtained reveal a substantial enhancement as the number of extra dimensions pro- 
jected onto the brane increases. The enhancement amounts to orders of magnitude, for large values of $n$ and for all particle species, although the increase in the rate depends strongly on the spin of the particle studied. The computed total relative emissivities reveal that scalar fields, which are the dominant form of particles emitted by the black hole for $n=0$, are outnumbered by the gauge bosons for large values of $n$, with the fermions being the least effective channel during the emission. Therefore, both the amount and the type of the emitted radiation by a higher-dimensional black hole directly on the brane may possibly lead to the determination of the number of extra dimensions.

The emission of particle modes on the brane is the most phenomenologically interesting effect since it involves Standard Model particles that can be easily detected during experiments. Nevertheless, a small higher-dimensional black hole emits also bulk modes and inevitably a proportion of the total energy is lost into the bulk. In the second part of this paper, we investigated the details of the emission of bulk scalar modes with the final aim being to provide an accurate estimate for the relative bulk-to-brane energy emissivity. The corresponding greybody factors depend again on the dimensionality of spacetime and so do the energy emission rates that exhibit a similar, although less substantial, enhancement as $n$ increases. Comparing the total bulk and brane emission rates for scalars, integrated over the whole energy regime, we conclude that most of the energy of the black hole is emitted in the form of brane modes for all values of $n$. The total emissivity in the bulk reduces to less than $1 / 4$ of that on the brane for $n=2,3,4$, while it becomes substantial for the extreme value of $n=7$, without however exceeding the brane value. The accurate results, produced here for the first time in the literature, clarify the situation concerning the relative amounts of energy emitted in the bulk and on the brane, and provide firm support to the heuristic arguments made in Ref. [27].

We should note at this point that no results were presented in this paper concerning the emission of gravitons either on the brane or in the bulk. The derivation of a consistent equation, that can describe the motion of gravitons on the induced black hole background on the brane, is still under investigation. Nevertheless, we expect that, as in the case of the emission of fields with spin $s=0,1$ and $\frac{1}{2}$, the graviton emission becomes enhanced as the dimensionality of spacetime increases, and that it remains subdominant compared to that of the other species at least for small values of $n$. In the near future, we hope to be able to present complete results for the emission rates for gravitons on the brane, and for the relative bulk-to-brane graviton emissivity.

A final comment needs to be made here concerning the validity of the results derived in this work. As pointed out in the text, the horizon of the black hole and its mass, or equivalently the energy needed for the production of the miniature black hole, is an input parameter of the analysis. Our results are applicable for all values of $r_{H}$ which are smaller than the size of the extra dimensions $R$, no matter how small 
or large $R$ is (as long as it remains considerably larger than $\ell_{P l}$ to avoid quantum corrections). Our analysis therefore remains valid for all theories postulating the existence of flat extra dimensions and a fundamental scale of gravity even a few orders of magnitude lower than $M_{P l}$. If the emission of Hawking radiation from these black holes is successfully detected, either at next-generation colliders or in the more distant future, the distinctive features discussed here may help in the determination of the number of extra dimensions that exist in nature.

\section{Acknowledgments}

We would like to thank John March-Russell for a constructive collaboration at an early stage, and Bryan Webber for helpful discussions and suggestions throughout this work. P.K. would also like to thank V. Rubakov, M. Shaposhnikov and P. Tinyakov for a stimulating discussion when this project had just begun, and R. Emparan for useful comments on the manuscript. C.H. was funded during this work by the U.K. Particle Physics and Astronomy Research Council.

\section{Appendix}

In this Appendix, we provide a few steps in the Newman-Penrose formalism for the derivation of the master equation describing the motion of a particle with spin $s$ in the background of a higher-dimensional, non-rotating, neutral black hole projected onto a 3-brane. The corresponding 4-dimensional metric tensor is given in Eq. (3.1). We first need to choose a tetrad basis of null vectors $\left(\ell^{\mu}, n^{\mu}, m^{\mu}, \bar{m}^{\mu}\right)$, where $\ell$ and $n$ are real vectors and $m$ and $\bar{m}$ are a pair of complex conjugate vectors. They satisfy the relations: $\mathbf{l} \cdot \mathbf{n}=1, \mathbf{m} \cdot \overline{\mathbf{m}}=-1$, with all other products being zero. Such a tetrad basis is given by:

$$
\begin{gathered}
\ell^{\mu}=\left(\frac{1}{h}, 1,0,0\right), \quad n^{\mu}=\left(\frac{1}{2},-\frac{h}{2}, 0,0\right), \\
m^{\mu}=\left(0,0,1, \frac{i}{\sin \theta}\right) \frac{1}{\sqrt{2} r}, \quad \bar{m}^{\mu}=\left(0,0,1, \frac{-i}{\sin \theta}\right) \frac{1}{\sqrt{2} r} .
\end{gathered}
$$

The $\lambda_{a b c}$ coefficients, which are used to construct the spin coefficients, are defined as: $\lambda_{a b c}=\left(e_{b}\right)_{i, j}\left[\left(e_{a}\right)^{i}\left(e_{c}\right)^{j}-\left(e_{a}\right)^{j}\left(e_{c}\right)^{i}\right]$, where $e_{a}$ stands for each one of the null vectors and $(i, j)$ denote the components of each vector. Their non-vanishing components are found to be:

$$
\lambda_{122}=-\frac{h^{\prime}}{2}, \quad \lambda_{134}=\frac{1}{r}, \quad \lambda_{234}=-\frac{h}{2 r}, \quad \lambda_{334}=\frac{\cos \theta}{\sqrt{2} r \sin \theta} .
$$

The above components must be supplemented by those that follow from the symmetry $\lambda_{a b c}=-\lambda_{c b a}$ and the complex conjugates obtained by replacing an index 3 by 4 (or vice versa) or interchanging 3 and 4 (when they are both present). 
We may now compute the spin coefficients defined by $\gamma_{a b c}=\left(\lambda_{a b c}+\lambda_{c a b}-\lambda_{b c a}\right) / 2$. Particular components, or combinations, of the spin coefficients can be directly used in the field equations $[35,36]$. They are found to have the values:

$$
\begin{gathered}
\kappa=\sigma=\lambda=\nu=\tau=\pi=\epsilon=0, \\
\rho=-\frac{1}{r}, \quad \mu=-\frac{h}{2 r}, \quad \gamma=\frac{h^{\prime}}{4}, \quad \alpha=-\beta=-\frac{\cot \theta}{2 \sqrt{2} r} .
\end{gathered}
$$

In what follows, we will also employ the Newman-Penrose operators:

$$
\hat{D}=\frac{1}{h} \frac{\partial}{\partial t}+\frac{\partial}{\partial r}, \quad \hat{\Delta}=\frac{1}{2} \frac{\partial}{\partial t}-\frac{h}{2} \frac{\partial}{\partial r}, \quad \hat{\delta}=\frac{1}{\sqrt{2} r}\left(\frac{\partial}{\partial \theta}+\frac{i}{\sin \theta} \frac{\partial}{\partial \varphi}\right),
$$

and make use of the following field factorization:

$$
\Psi_{s}(t, r, \theta, \varphi)=e^{-i \omega t} e^{i m \varphi} R_{s}(r) S_{s, \ell}^{m}(\theta),
$$

where $Y_{s, \ell}^{m}=e^{i m \varphi} S_{s, \ell}^{m}(\theta)$ are the spin-weighted spherical harmonics [37]. We will now consider each type of field separately:

\section{Gauge Bosons $(s=1)$}

In the Newman-Penrose formalism, there are only three 'degrees of freedom' for a gauge field, namely $\Phi_{0}=F_{13}, \Phi_{1}=\left(F_{12}+F_{43}\right) / 2$ and $\Phi_{2}=F_{42}$, in terms of which the different components of the Yang-Mills equation for a massless gauge field are written as:

$$
\begin{aligned}
(\hat{D}-2 \rho) \Phi_{1}-\left(\hat{\delta}^{*}-2 \alpha\right) \Phi_{0} & =0, \\
\hat{\delta} \Phi_{1}-(\hat{\Delta}+\mu-2 \gamma) \Phi_{0} & =0, \\
(\hat{D}-\rho) \Phi_{2}-\hat{\delta}^{*} \Phi_{1} & =0, \\
(\hat{\delta}+2 \beta) \Phi_{2}-(\hat{\Delta}+2 \mu) \Phi_{1} & =0,
\end{aligned}
$$

where $\hat{\delta}^{*}$ stands for the complex conjugate of $\hat{\delta}$. Rearranging Eqs. (6)-(7), one can see that $\Phi_{1}$ decouples leaving behind an equation involving only $\Phi_{0}$. Using the explicit forms of the operators and spin coefficients, as well as the factorized ansatz (5), this can be separated into an angular equation,

$$
\frac{1}{\sin \theta} \frac{d}{d \theta}\left(\sin \theta \frac{d S_{1, \ell}^{m}}{d \theta}\right)+\left[-\frac{2 m \cot \theta}{\sin \theta}-\frac{m^{2}}{\sin ^{2} \theta}+1-\cot ^{2} \theta+\lambda_{1 \ell}\right] S_{1, \ell}^{m}(\theta)=0,
$$

with eigenvalue $\lambda_{s \ell}=\ell(\ell+1)-s(s+1)$, and a radial equation:

$$
\frac{1}{\Delta} \frac{d}{d r}\left(\Delta^{2} \frac{d R_{1}}{d r}\right)+\left[\frac{\omega^{2} r^{2}}{h}+2 i \omega r-\frac{i \omega r^{2} h^{\prime}}{h}+\left(\Delta^{\prime \prime}-2\right)-\lambda_{1 \ell}\right] R_{1}(r)=0,
$$


where we have defined $\Delta=h r^{2}$.

\section{Fermion Fields $(s=1 / 2)$}

For a massless two-component spinor field, the Dirac equation can be written as:

$$
\begin{aligned}
\left(\hat{\delta}^{*}-\alpha\right) \chi_{0} & =(\hat{D}-\rho) \chi_{1}, \\
(\hat{\Delta}+\mu-\gamma) \chi_{0} & =(\hat{\delta}+\beta) \chi_{1} .
\end{aligned}
$$

Performing a similar rearrangement as in the case of bosons, we find that $\chi_{1}$ is decoupled and that the equation for $\chi_{0}$ reduces to the following set of angular,

$$
\frac{1}{\sin \theta} \frac{d}{d \theta}\left(\sin \theta \frac{d S_{1 / 2, \ell}^{m}}{d \theta}\right)+\left[-\frac{m \cot \theta}{\sin \theta}-\frac{m^{2}}{\sin ^{2} \theta}+\frac{1}{2}-\frac{1}{4} \cot ^{2} \theta+\lambda_{\frac{1}{2} \ell}\right] S_{1 / 2, \ell}^{m}(\theta)=0,
$$

and radial,

$$
\frac{1}{\sqrt{\Delta}} \frac{d}{d r}\left(\Delta^{3 / 2} \frac{d R_{1 / 2}}{d r}\right)+\left[\frac{\omega^{2} r^{2}}{h}+i \omega r-\frac{i \omega r^{2} h^{\prime}}{2 h}+\frac{1}{2}\left(\Delta^{\prime \prime}-2\right)-\lambda_{\frac{1}{2} \ell}\right] R_{1 / 2}(r)=0,
$$

equations, with the same definitions for $\Delta$ and $\lambda_{s \ell}$ as before.

\section{Scalar Fields $(s=0)$}

For completeness, we add here the equation of motion for a scalar field propagating in the same background. This equation can be determined quite easily by evaluating the double covariant derivative $g^{\mu \nu} D_{\mu} D_{\nu}$ acting on the scalar field. It finally leads to the pair of equations

$$
\begin{gathered}
\frac{1}{\sin \theta} \frac{d}{d \theta}\left(\sin \theta \frac{d S_{0, \ell}^{m}}{d \theta}\right)+\left[-\frac{m^{2}}{\sin ^{2} \theta}+\lambda_{0 \ell}\right] S_{0, \ell}^{m}=0, \\
\frac{d}{d r}\left(\Delta \frac{d R_{0}}{d r}\right)+\left(\frac{\omega^{2} r^{2}}{h}-\lambda_{0 \ell}\right) R_{0}(r)=0
\end{gathered}
$$

where $Y_{0, \ell}^{m}=e^{i m \varphi} S_{0, \ell}^{m}(\theta)$ are the usual spherical harmonics $Y_{\ell}^{m}(\theta, \varphi)$ and $\lambda_{0 \ell}=$ $\ell(\ell+1)$. The above equations were used in [25] for the analytic determination of the greybody factors for the emission of scalar particles on the brane by a higherdimensional black hole.

\section{$\underline{\text { IV. Master Equation for a field with arbitrary spin } s}$}

Combining all the above equations derived for bosons, fermions and scalar fields, we may now rewrite them in the form of a master equation valid for all types of fields. The radial equation then takes the form:

$$
\Delta^{-s} \frac{d}{d r}\left(\Delta^{s+1} \frac{d R_{s}}{d r}\right)+\left(\frac{\omega^{2} r^{2}}{h}+2 i s \omega r-\frac{i s \omega r^{2} h^{\prime}}{h}+s\left(\Delta^{\prime \prime}-2\right)-\lambda_{s \ell}\right) R_{s}(r)=0
$$


while, the angular equation reads

$$
\frac{1}{\sin \theta} \frac{d}{d \theta}\left(\sin \theta \frac{d S_{s, \ell}^{m}}{d \theta}\right)+\left[-\frac{2 m s \cot \theta}{\sin \theta}-\frac{m^{2}}{\sin ^{2} \theta}+s-s^{2} \cot ^{2} \theta+\lambda_{s \ell}\right] S_{s, \ell}^{m}=0 .
$$

The latter equation is identical to the one derived by Teukolsky [34] in the case of a non-rotating, spherically-symmetric black hole. The radial one differs by the extra factor $s\left(\Delta^{\prime \prime}-2\right)$ due to the fact that for our metric tensor this combination is not zero, contrary to what happens in the case of the 4-dimensional Schwarzschild, or Kerr, metric. The $\Delta^{\prime \prime}$ - term can be removed if we make the redefinition $R_{s}=\Delta^{-s} P_{s}$. Then, we obtain:

$$
\Delta^{s} \frac{d}{d r}\left(\Delta^{1-s} \frac{d P_{s}}{d r}\right)+\left(\frac{\omega^{2} r^{2}}{h}+2 i s \omega r-\frac{i s \omega r^{2} h^{\prime}}{h}-\Lambda\right) P_{s}(r)=0,
$$

where now $\Lambda=\lambda_{s \ell}+2 s=\ell(\ell+1)-s(s-1)$. The above form of the radial equation was used in [26] to determine in an analytic way the greybody factors and emission rates for the emission of fermions and gauge bosons on the brane by a higher-dimensional, spherically-symmetric black hole.

\section{References}

[1] N. Arkani-Hamed, S. Dimopoulos and G. Dvali, The Hierarchy Problem and New Dimensions at a Millimeter, Phys. Lett. B 429 (1998) 263 hep-ph/9803315;

N. Arkani-Hamed, S. Dimopoulos and G. Dvali, Phenomenology, astrophysics and cosmology of theories with sub-millimeter dimensions and TeV scale quantum gravity, Phys. Rev. D 59 (1999) 086004 hep-ph/9807344;

I. Antoniadis, N. Arkani-Hamed, S. Dimopoulos and G. R. Dvali, New dimensions at a millimeter to a Fermi and superstrings at a TeV, Phys. Lett. B 436 (1998) 257 hep-ph/9804398.

[2] L. Randall and R. Sundrum, A Large Mass Hierarchy from a Small Extra Dimension, Phys. Rev. Lett. 83 (1999) 3370 hep-ph/9905221;

L. Randall and R. Sundrum, An alternative to compactification, Phys. Rev. Lett. 83 (1999) 4690 hep-th/9906064.

[3] K. Akama, An Early Proposal Of 'Brane World', Lect. Notes Phys. 176 (1982) 267 hep-th/0001113;

V. A. Rubakov and M. E. Shaposhnikov, Extra spacetime Dimensions: Towards A Solution To The Cosmological Constant Problem, Phys. Lett. B 125 (1983) 139;

V. A. Rubakov and M. E. Shaposhnikov, Do We Live Inside A Domain Wall?, Phys. Lett. B 125 (1983) 136;

M. Visser, An Exotic Class Of Kaluza-Klein Models, Phys. Lett. B 159 (1985) 22 hep-th/9910093; 
I. Antoniadis, A Possible New Dimension At A Few Tev, Phys. Lett. B 246 (1990) 377 ;

I. Antoniadis, K. Benakli and M. Quiros, Production of Kaluza-Klein states at future colliders, Phys. Lett. B 331 (1994) 313 hep-ph/9403290;

J. Lykken, Weak Scale Superstrings, Phys. Rev. D 54 (1996) 3693 hep-th/9603133.

[4] C. D. Hoyle, U. Schmidt, B. R. Heckel, E. G. Adelberger, J. H. Gundlach, D. J. Kapner and H. E. Swanson, Sub-millimeter tests of the gravitational inverse-square law: A search for 'large' extra dimensions, Phys. Rev. Lett. 86 (2001) 1418 hep-ph/0011014.

[5] P. Abreu et al. [DELPHI Collaboration], Photon Events with Missing Energy at $\sqrt{s}=$ 183 to 189 GeV, Eur. Phys. J. C 17 (2000) 53 hep-ex/0103044;

G. Abbiendi et al. [OPAL Collaboration], Photonic events with missing energy in e+ $e$ collisions at $s^{* *}(1 / 2)=189-G e V$," Eur. Phys. J. C 18 (2000) 253 hep-ex/0005002;

D. Acosta et al. [CDF Collaboration], Limits on extra dimensions and new particle production in the exclusive photon and missing energy signature in $p$ anti-p collisions at $s^{* *}(1 / 2)=1.8-\mathrm{TeV}$, Phys. Rev. Lett. 89 (2002) 281801 hep-ex/0205057.

[6] S. Cullen and M. Perelstein, SN1987A constraints on large compact dimensions, Phys. Rev. Lett. 83 (1999) 268 hep-ph/9903422];

L. J. Hall and D. R. Smith, Cosmological constraints on theories with large extra dimensions, Phys. Rev. D 60 (1999) 085008 hep-ph/9904267;

V. D. Barger, T. Han, C. Kao and R. J. Zhang, Astrophysical constraints on large extra dimensions, Phys. Lett. B 461 (1999) 34 hep-ph/9905474;

C. Hanhart, D. Phillips, S. Reddy and M. Savage, Extra dimensions, SN1987a, and nucleon nucleon scattering data, Nucl. Phys. B 595 (2001) 335 nucl-th/0007016;

C. Hanhart, J. A. Pons, D. R. Phillips and S. Reddy, The likelihood of GODs' existence: Improving the SN 1987 a constraint on the size of large compact dimensions, Phys. Lett. B 509 (2001) 1 astro-ph/0102063;

S. Hannestad, Strong constraint on large extra dimensions from cosmology, Phys. Rev. D 64 (2001) 023515 hep-ph/0102290;

S. Hannestad and G. Raffelt, New supernova limit on large extra dimensions, Phys. Rev. Lett. 87 (2001) 051301 hep-ph/0103201;

S. Hannestad and G. G. Raffelt, Stringent neutron-star limits on large extra dimensions, Phys. Rev. Lett. 88 (2002) 071301 hep-ph/0110067;

R. Allahverdi, C. Bird, S. Groot Nibbelink and M. Pospelov, Cosmological bounds on large extra dimensions from non-thermal production of Kaluza-Klein modes, hep-ph/0305010;

L. A. Anchordoqui, J. L. Feng, H. Goldberg and A. D. Shapere, Updated limits on TeV-scale gravity from absence of neutrino cosmic ray showers mediated by black holes, hep-ph/0307228. 
[7] P. C. Argyres, S. Dimopoulos and J. March-Russell, Black holes and sub-millimeter dimensions, Phys. Lett. B 441 (1998) 96 hep-th/9808138].

[8] T. Banks and W. Fischler, hep-th/9906038.

[9] S. B. Giddings and S. Thomas, High Energy Colliders as Black Hole Factories: The End of Short Distance Physics, Phys. Rev. D 65 (2002) 056010 hep-ph/0106219.

[10] S. Dimopoulos and G. Landsberg, Black holes at the LHC, Phys. Rev. Lett. 87 (2001) 161602 hep-ph/0106295.

[11] M. B. Voloshin, Semiclassical suppression of black hole production in particle collisions, Phys. Lett. B 518 (2001) 137 hep-ph/0107119;

M. B. Voloshin, More Remarks on Suppression of Large Black Hole Production in Particle Collisions, Phys. Lett. B 524 (2002) 376 hep-ph/0111099.

[12] S. Dimopoulos and R. Emparan, String balls at the LHC and beyond, Phys. Lett. B 526 (2002) 393 hep-ph/0108060.

[13] S. B. Giddings, Black Hole Production in TeV-scale Gravity, and the Future of High Energy Physics, hep-ph/0110127.

[14] G. F. Giudice, R. Rattazzi and J. D. Wells, Transplanckian collisions at the LHC and beyond, Nucl. Phys. B 630 (2002) 293 hep-ph/0112161.

[15] K. S. Thorne, Nonspherical Gravitational Collapse: A Short Review, in Magic without Magic, Ed. J. R. Klauder (San Fransisco, 1972).

[16] R. Penrose, unpublished, 1974;

P. D. D'Eath and P. N. Payne, Gravitational Radiation In High Speed Black Hole Collisions. 1. Perturbation Treatment Of The Axisymmetric Speed Of Light Collision, Phys. Rev. D 46 (1992) 658;

D. M. Eardley and S. B. Giddings, Classical Black Hole Production in High-Energy Collisions, Phys. Rev. D 66 (2002) 044011 gr-qc/0201034;

H. Yoshino and Y. Nambu, High-energy head-on collisions of particles and hoop conjecture, Phys. Rev. D 66 (2002) 065004 gr-qc/0204060.

[17] A. Goyal, A. Gupta and N. Mahajan, Neutrinos as source of ultra high-energy cosmic rays in extra dimensions, Phys. Rev. D 63 (2001) 043003 hep-ph/0005030;

J. L. Feng and A. D. Shapere, Black hole production by cosmic rays, Phys. Rev. Lett. 88 (2002) 021303 hep-ph/0109106;

L. Anchordoqui and H. Goldberg, Experimental signature for black hole production in neutrino air showers, Phys. Rev. D 65 (2002) 047502 hep-ph/0109242;

R. Emparan, M. Masip and R. Rattazzi, Cosmic rays as probes of large extra dimensions and TeV gravity, Phys. Rev. D 65 (2002) 064023 hep-ph/0109287;

L. A. Anchordoqui, J. L. Feng, H. Goldberg and A. D. Shapere, Black holes from 
cosmic rays: Probes of extra dimensions and new limits on TeV-scale gravity, Phys. Rev. D 65 (2002) 124027 hep-ph/0112247;

Y. Uehara, Production and detection of black holes at neutrino array, Prog. Theor. Phys. 107 (2002) 621 [hep-ph/0110382;

J. Alvarez-Muniz, J. L. Feng, F. Halzen, T. Han and D. Hooper, Detecting microscopic black holes with neutrino telescopes, Phys. Rev. D 65 (2002) 124015 hep-ph/0202081.

[18] A. Ringwald and H. Tu, Collider versus cosmic ray sensitivity to black hole production, Phys. Lett. B 525 (2002) 135 hep-ph/0111042;

M. Kowalski, A. Ringwald and H. Tu, Black holes at neutrino telescopes, Phys. Lett. B 529 (2002) 1 hep-ph/0201139;

D. Kazanas and A. Nicolaidis, Cosmic rays and large extra dimensions, Gen. Rel. Grav. 35 (2003) 1117 [hep-ph/0109247];

P. Jain, S. Kar, S. Panda and J. P. Ralston, Brane-production and the neutrino nucleon cross section at ultra high energies in low scale gravity models, hep-ph/0201232;

A. Ringwald, Production of black holes in TeV-scale gravity, Fortsch. Phys. 51 (2003) 830 hep-ph/0212342;

E. J. Ahn, M. Ave, M. Cavaglia and A. V. Olinto, TeV black hole fragmentation and detectability in extensive air-showers, Phys. Rev. D 68 (2003) 043004 hep-ph/0306008;

A. Nicolaidis and N. G. Sanchez, Signatures of TeV scale gravity in high energy collisions, hep-ph/0307321;

L. A. Anchordoqui, J. L. Feng, H. Goldberg and A. D. Shapere, Updated limits on TeV-scale gravity from absence of neutrino cosmic ray showers mediated by black holes, hep-ph/0307228.

[19] S. Hossenfelder, S. Hofmann, M. Bleicher and H. Stocker, Quasi-stable black holes at LHC, Phys. Rev. D 66 (101502) 2002 hep-ph/0109085;

H. C. Kim, S. H. Moon and J. H. Yee, J. High Energy Phys. 0202 (2002) 046 gr-qc/0108071;

K. Cheung, Black hole production and large extra dimensions, Phys. Rev. Lett. 88 (2002) 221602 hep-ph/0110163;

S. Hossenfelder, S. Hofmann, M. Bleicher and H. Stocker, Black hole production in large extra dimensions at the Tevatron: A chance to observe a first glimpse of TeV scale gravity, Phys. Lett. B 548 (200) 73 hep-ph/0112186;

R. Casadio and B. Harms, Can black holes and naked singularities be detected in accelerators?, Int. J. Mod. Phys. A 17 (2002) 4635 hep-th/0110255;

S. C. Park and H. S. Song, Production of spinning black holes at colliders, J. Korean Phys. Soc. 43 (2003) 30 hep-ph/0111069;

G. Landsberg, Discovering new physics in the decays of black holes, Phys. Rev. Lett. 88 (2002) 181801 hep-ph/0112061. 
[20] E. J. Ahn, M. Cavaglia and A. V. Olinto, Brane factories, Phys. Lett. B 551 (2003) 1 hep-th/0201042;

T. G. Rizzo, Black hole production at the LHC: Effects of Voloshin suppression, J. High Energy Phys. 0202 (2002) 011 [hep-ph/0201228;

S. N. Solodukhin, Classical and quantum cross-section for black hole production in particle collisions, Phys. Lett. B 533 (2002) 153 hep-ph/0201248;

V. Cardoso and J. P. Lemos, Gravitational radiation from collisions at the speed of light: A massless particle falling into a Schwarzschild black hole, Phys. Lett. B 538 (2002) 1 gr-qc/0202019;

K. Cheung, Black hole, string ball, and p-brane production at hadronic supercolliders, Phys. Rev. D 66 (2002) 036007 hep-ph/0205033;

R. A. Konoplya, On quasinormal modes of small Schwarzschild-anti-de-Sitter black hole, Phys. Rev. D 66 (2002) 044009 hep-th/0205142];

Y. Uehara, New potential of black holes: Quest for TeV-scale physics by measuring top quark sector using black holes, hep-ph/0205199;

R. Guedens, D. Clancy and A. R. Liddle, Primordial black holes in braneworld cosmologies. I: Formation, cosmological evolution and evaporation, Phys. Rev. D 66 (2002) 043513 astro-ph/0205149.

[21] A. V. Kotwal and C. Hays, Production and decay of spinning black holes at colliders and tests of black hole dynamics, Phys. Rev. D 66 (2002) 116005 hep-ph/0206055;

V. Frolov and D. Stojkovic, Black hole radiation in the brane world and recoil effect, Phys. Rev. D 66 (2002) 084002 hep-th/0206046;

A. Chamblin and G. C. Nayak, Black hole production at LHC: String balls and black holes from pp and lead lead collisions, Phys. Rev. D 66 (2002) 091901 hep-ph/0206060;

T. Han, G. D. Kribs and B. McElrath, Black hole evaporation with separated fermions, Phys. Rev. Lett. 90 (2003) 031601 hep-ph/0207003;

V. Frolov and D. Stojkovic, Black hole as a point radiator and recoil effect on the brane world, Phys. Rev. Lett. 89 (2002) 151302 hep-th/0208102;

L. Anchordoqui and H. Goldberg, Black hole chromosphere at the LHC, Phys. Rev. D 67 (2003) 064010 hep-ph/0209337.

[22] V. Frolov and D. Stojkovic, Quantum radiation from a 5-dimensional rotating black hole, Phys. Rev. D 67 (2003) 084004 gr-qc/0211055;

V. Cardoso and J. P. Lemos, Gravitational radiation from the radial infall of highly relativistic point particles into Kerr black holes, Phys. Rev. D 67 (2003) 084005 gr-qc/0211094;

V. Cardoso, O. J. Dias and J. P. Lemos, Gravitational radiation in D-dimensional spacetimes, Phys. Rev. D 67 (2003) 064026 hep-th/0212168;

V. Frolov and D. Stojkovic, Particle and light motion in a space-time of a five- 
dimensional rotating black hole, gr-qc/0301016;

A. Chamblin, F. Cooper and G. C. Nayak, Interaction of a TeV scale black hole with the quark gluon plasma at LHC, hep-ph/0301239;

I. Mocioiu, Y. Nara and I. Sarcevic, Hadrons as signature of black hole production at the LHC, Phys. Lett. B 557 (2003) 87 hep-ph/0301073;

R. A. Konoplya, Quasinormal behavior of the d-dimensional Schwarzshild black hole and higher order WKB approach, Phys. Rev. D 68 (2003) 024018 [gr-qc/0303052];

R. Casadio, On brane-world black holes and short scale physics, hep-ph/0304099;

O. Vasilenko, Trap surface formation in high-energy black holes collision, hep-th/0305067;

M. Cavaglia, S. Das and R. Maartens, Will we observe black holes at LHC?, Class. and Quant. Grav. 20 (2003) L205 hep-ph/0305223;

R. A. Konoplya, Gravitational quasinormal radiation of higher-dimensional black holes, hep-th/0309030.

[23] S. W. Hawking, Particle Creation by Black Holes, Comm. Math. Phys. 43 (1975) 199.

[24] D. N. Page Particle Emission Rates from a Black Hole: Massless Particles from an Uncharged, Nonrotating Hole, Phys. Rev. D 13 (1976) 198.

[25] P. Kanti and J. March-Russell, Calculable Corrections to Brane Black Hole Decay. 1. The Scalar Case, Phys. Rev. D 66 (2002) 024023 hep-ph/0203223].

[26] P. Kanti and J. March-Russell, Calculable Corrections to Brane Black Hole Decay. 2. Greybody Factors for Spin 1/2 and 1, Phys. Rev. D 67 (2003) 104019 hep-ph/0212199.

[27] R. Emparan, G. T. Horowitz and R. C. Myers, Black Holes Radiate Mainly on the Brane, Phys. Rev. Lett. 85 (2000) 499 hep-th/0003118].

[28] R. C. Myers and M. J. Perry, Black Holes in Higher Dimensional spacetimes, Ann. Phys. (NY) 172 (1986) 304.

[29] S. S. Gubser, I. R. Klebanov and A. A. Tseytlin, String theory and classical absorption by three-branes, Nucl. Phys. B 499 (1997) 217 hep-th/9703040.

[30] C. M. Harris, P. Richardson and B. R. Webber, "CHARYBDIS: A black hole event generator, J. High Energy Phys. 0308 (2003) 033 hep-ph/0307305.

[31] C. W. Misner, K. T. Thorne and J. A. Wheeler, Gravitation (Freeman, San Fransisco, 1973).

[32] N. Sanchez, Absorption And Emission Spectra Of A Schwarzschild Black Hole, Phys. Rev. D 18 (1978) 1030;

N. Sanchez, Elastic Scattering of Waves by a Black Hole, Phys. Rev. D 18 (1978) 1798 . 
[33] J. H. MacGibbon and B. R. Webber, Quark- and Gluon-jet Emission from Primordial Black Holes: The Instantaneous Spectra, Phys. Rev. D 41 (1990) 3052.

[34] S. A. Teukolsky, Perturbations of a Rotating Black Hole. I. Fundamental Equations for Gravitational, Electromagnetic and Neutrino-Field Perturbations, Astrophys. J. 185 (1973) 635.

[35] E. Newman and R. Penrose, An approach to Gravitational Radiation by a Method of Spin Coefficients, J. Math. Phys. 3 (1962) 566.

[36] S. Chandrasekhar, The Mathematical Theory of Black Holes (Oxford University Press, New York, 1983).

[37] J. N. Goldberg, A. J. MacFarlane, E. T. Newman, F. Rohrlich and E. C. Sudarshan, Spin S Spherical Harmonics And Edth, J. Math. Phys. 8 (1967) 2155.

[38] M. Cvetic and F. Larsen, Greybody factors for black holes in four dimensions: Particles with spin, Phys. Rev. D 57 (1998) 6297 hep-th/9712118.

[39] D. Ida, K-y. Oda and S. C. Park, Rotating Black Holes at Future Colliders: Greybody Factors for Brane Fields, Phys. Rev. D 67 (2003) 064026 hep-th/0212108.

[40] W. H. Press and S. A. Teukolsky, Perturbations of a Rotating Black Hole. III. Interaction of the Hole with Gravitational and Electromagnetic Radiation, Astrophys. J. 193 (1974) 443.

[41] M. Cavaglia, Black hole multiplicity at particle colliders (Do black holes radiate mainly on the brane?), hep-ph/0305256. 